\title{
Outage in Large Wireless Networks with Spectrum Sharing under Rayleigh Fading
}

by

Arshdeep S. Kahlon, B.E.

A thesis submitted to The Faculty of Graduate and Postdoctoral Affairs in partial fulfillment of the requirements for the degree of

Masters of Applied Science in Electrical and Computer Engineering

Ottawa-Carleton Institute for Electrical and Computer Engineering (OCIECE)

Department of Systems and Computer Engineering

Carleton University

Ottawa, Ontario, Canada, K1S 5B6

June 2011

(C)Copyright 2011, Arshdeep S. Kahlon 
Library and Archives

Canada

Published Heritage

Branch

395 Wellington Street

Ottawa ON K1A ON4

Canada
Bibliothèque et

Archives Canada

Direction du

Patrimoine de l'édition

395 , rue Wellington

Ottawa ON K1A ON4

Canada
Your file Votre référence
ISBN: $978-0-494-83034-5$
Our file Notre référence
ISBN: $978-0-494-83034-5$
NOTICE:

The author has granted a nonexclusive license allowing Library and Archives Canada to reproduce, publish, archive, preserve, conserve, communicate to the public by telecommunication or on the Internet, loan, distribute and sell theses worldwide, for commercial or noncommercial purposes, in microform, paper, electronic and/or any other formats.

The author retains copyright ownership and moral rights in this thesis. Neither the thesis nor substantial extracts from it may be printed or otherwise reproduced without the author's permission.
AVIS:

L'auteur a accordé une licence non exclusive permettant à la Bibliothèque et Archives Canada de reproduire, publier, archiver, sauvegarder, conserver, transmettre au public par télécommunication ou par l'Internet, prêter, distribuer et vendre des thèses partout dans le monde, à des fins commerciales ou autres, sur support microforme, papier, électronique et/ou autres formats.

L'auteur conserve la propriété du droit d'auteur et des droits moraux qui protège cette thèse. Ni la thèse ni des extraits substantiels de celle-ci ne doivent être imprimés ou autrement reproduits sans son autorisation.
In compliance with the Canadian Privacy Act some supporting forms may have been removed from this thesis.

While these forms may be included in the document page count, their removal does not represent any loss of content from the thesis.
Conformément à la loi canadienne sur la protection de la vie privée, quelques formulaires secondaires ont été enlevés de cette thèse.

Bien que ces formulaires aient inclus dans la pagination, il n'y aura aucun contenu manquant.

\section{Canadä}


The undersigned recommend to The Faculty of Graduate and Postdoctoral Affairs acceptance of the thesis

\section{Outage in Large Wireless Networks with Spectrum Sharing under Rayleigh Fading}

submitted by

Arshdeep S. Kahlon

in partial fulfillment of the requirements for the degree of

Masters of Applied Science in Electrical and Computer Engineering

Thesis Co-supervisor

Professor Shalini Periyalwar

Thesis Co-supervisor

Professor Halim Yanikomeroglu

Chair, Department of Systems and Computer Engineering

Professor Howard M. Schwartz

Carleton University

June 2011 


\section{Abstract}

Outage experienced by a user in an incumbent primary network due to a secondary network deployment plays a key role in determining the spectrum sharing gains. While it is impossible to deterministically study large networks, which are characterized by the uncertainty in the number and the locations of the nodes, point process theory has proved to be a useful mathematical tool in studying the statistical behaviour of such networks.

The outage analysis, which is dependent on the sum of all the unwanted signals at the primary receiver, requires the study of the interference coming from the primary network as well. While analytical results are not tractable for complex primary networks, an alternative way to approach this problem in a Rayleigh-faded environment is provided in this thesis. A simple, yet widely applicable, expression for the outage due to two independent unwanted signal sets is derived. This expression does not require the knowledge of the distribution of net unwanted signal from one of these sets provided that the outage resulting due to this distribution is known. The spectrum sharing gains and opportunities for a finite field secondary network are then analyzed using closed-form expressions. As an application, a spectrum sharing scenario between the overlaid cellular and ad hoc networks is considered for the uplink case, and the outage is analyzed at the randomly, not necessarily independently, distributed cellular network receivers. Additionally, a tight upper bound on the outage is derived by incorporating a sensing mechanism to alleviate the profound effect of nearby secondary transmissions. It is worth mentioning that other than the spectrum sharing scenarios, the results presented are applicable for any set of interferers distributed (in the same or different networks) around any receiver of study. 


\section{Acknowledgements}

I would say this time period has been the best academic time in my life, so far and the credit goes to both my supervisors: Prof. Shalini Periyalwar and Prof. Halim Yanikomeroglu. I could not have wished for better supervisors. I feel honored to be a member of such an elite research group. The inspirational group meetings conducted on a regular basis not only gave me rewarding suggestions but also groomed my skills. Thanks to the whole group for their cooperation and improving my work.

Special thanks to Talha Ahmad for sitting in a chair next to me and always being such a supportive person. Thanks to Yaser Fouad, Amaninder Saini, Aizaz Chaudhary and Nazia Ahmad without whom my stay at Carleton would not have been so enjoyable. I would like to thank Dr. Muhammad Aljuaid for his sincere guidance and introducing me to the stochastic geometry. I would like to express my gratitude to Dr. Sebastian Szyszkowicz whom I have had the opportunity to work with. It was a true previlege to have fruitful discussions with him.

I am thankful to Carleton University including Department of Systems and Computer Engineering, for giving me an excellent platform to pursue my masters. I am grateful to the technical staff for handling computer related problems on time.

Finally, I am thankful to my parents for their persistent support and unconditional love. I feel fortunate to have such a wonderful sister, Pawanpreet, who is always there to encourage me. I am lucky to have cute nephews: Gurbir and Anantbir, who at times act as stress busters to me. 


\section{Contents}

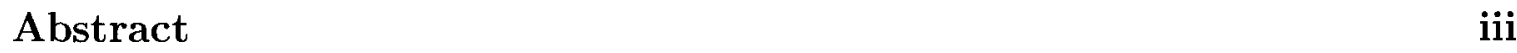

Acknowledgements $\quad$ iv

$\begin{array}{lll}\text { Contents } & \text { v }\end{array}$

List of Figures $\quad$ vii

Nomenclature $\quad$ ix

1 Introduction $\quad 1$

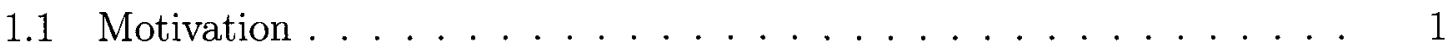

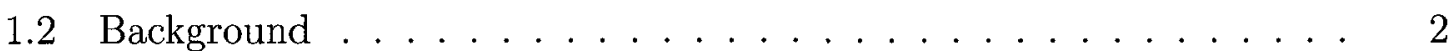

1.3 Mathematical Preliminaries . . . . . . . . . . . . 6

1.3.1 Poisson Point Process . . . . . . . . . . . . . . 7

1.3.2 Laplace Functional . . . . . . . . . . . . . . . . . . . 7

1.3.3 Marked Poisson Point Process . . . . . . . . . . . . . 8

1.4 Thesis Contribution . .................. 8

1.5 Thesis Structure . . . . . . . . . . . . . . . . 11

1.6 Manuscripts Accepted, Submitted, and In Progress . . . . . . . . . 11

2 Outage due to Independent Unwanted Signals $\quad 13$

2.1 Introduction . . . . . . . . . . . . . . . . 13 
2.2 Two Independent Unwanted Signals . . . . . . . . . . . . . . . . . . 14

2.3 Outage Analysis . . . . . . . . . . . . . . . . . . . . . . 15

2.4 Significance of the Derived Result . . . . . . . . . . . . 16

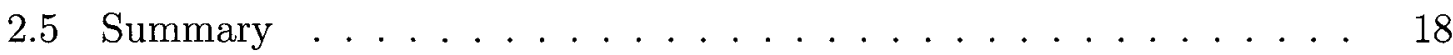

3 Spectrum Sharing for Finite Field Secondary Network 19

3.1 Introduction . . . . . . . . . . . . . . . . . . . . 19

3.2 System Model . . . . . . . . . . . . . . . . . . . . . . . 22

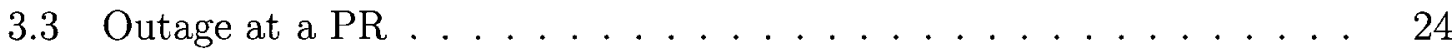

3.3.1 Effect of Field Size on the Spectrum Sharing Opportunities . . 29

3.3.2 Deployment of Maximum Number of STs . . . . . . . . . . 32

3.3.3 Bounded and Unbounded Path-loss models . . . . . . . . . . . 34

3.4 Summary . . . . . . . . . . . . . . . . . 37

4 Cellular Network Overlaid by an Ad hoc Network Incorporating the Sensing Mechanism: The Uplink Case $\quad 39$

4.1 Introduction . . . . . . . . . . . . . . . . . 39

4.2 System Model . . . . . . . . . . . . . . . . . . . . . . . . 41

4.3 Outage Analysis . . . . . . . . . . . . . . . . . . . 43

4.3.1 Without Sensing Mechanism . . . . . . . . . . . . 43

4.3.2 With Sensing Mechanism . . . . . . . . . . . . . 44

4.3.3 Optimum Decision Threshold . . . . . . . . . . . . . . 48

4.4 Comparison between Presumably Exact and Soft Exclusion Regions . 50

4.5 Summary . . . . . . . . . . . . . . . . . . . 54

5 Conclusion and Future Work $\quad 55$

5.1 Thesis Achievements . . . . . . . . . . . . . . 55

5.2 Future Work . . . . . . . . . . . . . . . . . 57 


\section{List of Figures}

3.1 Set of STs distributed as a PPP over some general region. . . . . . . 25

3.2 STs distributed over a sector around the PR. . . . . . . . . . . . . 27

3.3 Maximum density of STs $\left(\lambda_{\max }\right)$ vs. additional outage constraint $\left(\Delta \varepsilon_{1, \mathrm{th}}\right)$. Lines correspond to analysis, while circles represent simulation with $r_{1}=10 \mathrm{~m}, r_{2}=50 \mathrm{~m}, \theta=\pi / 3, \alpha=3, \rho_{1, \mathrm{r}}=1 \mu \mathrm{W}, \beta_{1}=10$, and $\rho_{\mathrm{t}}=0.1 \mathrm{~mW}$.

3.4 Additional outage $\left(\Delta \varepsilon_{1}\right)$ vs. outer field radius $\left(r_{2}\right)$. Lines correspond to analysis, while circles represent simulation, with $r_{1}=20 \mathrm{~m}$, $\lambda=0.003 / \mathrm{m}^{2}, \theta=2 \pi, \varepsilon_{1}=0.4, \Delta \varepsilon_{1, \mathrm{th}}=0.35, \alpha=3, \rho_{1, \mathrm{r}}=1 \mu \mathrm{W}, \beta_{1}=10$, and $\rho_{\mathrm{t}}=0.1 \mathrm{~mW} \ldots \ldots \ldots \ldots \ldots \ldots$

3.5 Total outage $\varepsilon_{1, \mathrm{~T}}$ vs. inner field radius or exclusion region $r_{1}$ for different outer radius $r_{2}$, with $\lambda=0.003 / \mathrm{m}^{2}, \varepsilon_{1}=0.4, \alpha=3, \rho_{1, \mathrm{r}}=1 \mu \mathrm{W}, \beta_{1}=10$, $\rho_{\mathrm{t}}=0.1 \mathrm{~mW} \ldots \ldots \ldots \ldots \ldots \ldots$

3.6 Maximum average number of STs $\left(N_{\max }\right)$ vs. field radii $\left(r_{1}, r_{2}\right)$, with $\lambda=0.003 / \mathrm{m}^{2}, \varepsilon_{1}=0.4, \Delta \varepsilon_{1, \mathrm{th}}=0.35, \alpha=3, \rho_{1, \mathrm{r}}=1 \mu \mathrm{W}, \beta_{1}=10, \rho_{\mathrm{t}}=0.1 \mathrm{~mW}$. Shaded region corresponds to infeasible region, i.e., $r_{1} \geq r_{2} \ldots \ldots$.

3.7 Total outage $\left(\varepsilon_{1, \mathrm{~T}}\right)$ vs. ST transmit power $\rho_{\mathrm{t}}$, with simulation parameters: $r_{1}=10 \mathrm{~m}, r_{2}=1000 \mathrm{~m}, \theta=\pi / 3, \alpha=3, \rho_{1, \mathrm{r}}=1 \mu \mathrm{W}, \beta_{1}=10, \lambda=$

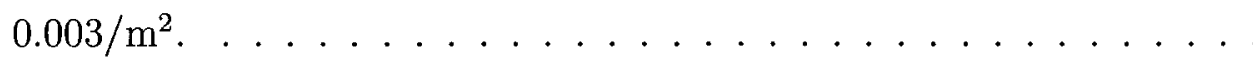


3.8 Percentage increase in total outage $\left(\delta \varepsilon_{1, \mathrm{~T}}\right)$ vs. field radius $r_{2}$, when unbounded path-loss model is used over bounded path-loss model, for different path-loss exponent $\alpha$ with simulation parameters $r_{0}=1 \mathrm{~m}$, $\theta=2 \pi, \rho_{1, \mathrm{r}}=1 \mu \mathrm{W}, C_{0}=1, \rho_{\mathrm{t}}=0.1 \mathrm{~mW}, \beta=10, \lambda=0.003 / \mathrm{m}^{2} . \ldots$

3.9 Percentage increase in total outage $\left(\delta \varepsilon_{1, \mathrm{~T}}\right)$ vs. density of STs $\lambda$, when unbounded path-loss model is used over bounded path-loss model, for different field-size $r_{2}$ with simulation parameters $r_{0}=1 \mathrm{~m}, \alpha=3, \theta=2 \pi$, $\rho_{1, r}=1 \mathrm{pW}, C_{0}=1, \rho_{\mathrm{t}}=0.1 \mathrm{~mW}, \beta_{1}=10 \ldots \ldots \ldots \ldots$

4.1 Illustration of the cellular network sharing spectrum with the secondary ad hoc network in the uplink case. . . . . . . . . . . . . .

4.2 Total outage $\varepsilon_{2, \mathrm{t}}$ suffered by the $i^{\text {th }} \mathrm{PR}$ with the density of STs $\lambda$ for $\varepsilon_{\imath}=0.05, \alpha=3, \rho_{\imath, \mathrm{r}}=1 \mu \mathrm{W}, \beta_{\imath}=10, \rho_{\mathrm{t}}=1 \mathrm{~mW}, \rho_{\mathrm{b}}=1 \mathrm{~W}$, and $\beta_{\imath, \mathrm{th}}=10$. Distance between the adjacent co-channel BSs is taken as $500 \mathrm{~m}$. . . .

4.3 Total outage $\varepsilon_{\imath, \mathrm{T}}$ suffered by the $i^{\text {th }} \mathrm{PR}$ with the decision threshold $\beta_{\imath, \mathrm{th}}=\beta_{\mathrm{th}}$, for $\lambda=10^{-4} / \mathrm{m}^{2}, \varepsilon_{\imath}=0.05, \alpha=3, \rho_{\imath, \mathrm{r}}=1 \mu \mathrm{W}, \beta_{\imath}=10, \rho_{\mathrm{b}}=1$, and $\rho_{\mathrm{t}}=1 \mathrm{~mW}$. Distance between the adjacent BSs is taken as $500 \mathrm{~m}$.

4.4 Outage difference $\delta \varepsilon_{\mathrm{T}}$ with the field-size $r_{2}$ for different density $\lambda$ values of STs, $\alpha=3, \varepsilon_{1}=0.05, \rho_{\mathrm{t}}=1 \mathrm{~mW}, \beta_{\mathrm{th}}=10$, and $\rho_{\mathrm{b}}=1 \mathrm{~W} \ldots \ldots \ldots$

4.5 Outage difference $\delta \varepsilon_{\mathrm{T}}$ with the density $\lambda$ of STs for different field-size $r_{2}, \alpha=3, \varepsilon_{1}=0.05, \rho_{\mathrm{t}}=1 \mathrm{~mW}, \beta_{\mathrm{th}}=10$, and $\rho_{\mathrm{b}}=1 \mathrm{~W} . \ldots \ldots$

4.6 CCDF of the number of ST nodes in case of presumably exact exclusion region $N_{1}$ and the soft exclusion region (formed by considering fading on the sensing channel) $N_{2}$, for $r_{2}=100, \eta=10^{-6}, \alpha=3, \beta_{\text {th }}=10$, and $\rho_{\mathrm{b}}=1 \mathrm{~W} \ldots \ldots \ldots \ldots \ldots \ldots \ldots \ldots \ldots \ldots \ldots \ldots \ldots \ldots \ldots$ 


\section{Nomenclature}

\section{Acronyms}

\begin{tabular}{ll}
\hline Acronym & Meaning \\
\hline \hline CDMA & Code division multiple access \\
CCDF & Complementary Cumulative Distribution Function \\
HPPP & Homogenous Poisson point process \\
PN & Primary network \\
PPP & Poisson point process \\
PR & Primary receiver \\
PT & Primary transmitter \\
PU & Primary user \\
SN & Secondary network \\
SU & Secondary user \\
ST & Secondary transmitter \\
SINR & Signal-to-interference-plus-noise ratio \\
SIR & Signal-to-interference ratio \\
SNR & Signal-to-noise ratio \\
\hline
\end{tabular}




\section{Mathematical Operators and Symbols}

\begin{tabular}{ll}
\hline Notation & Meaning \\
\hline \hline $\mathbb{E}(X)$ & Expected value of random variable $X$ \\
$\mathcal{P}(\mathcal{E})$ & Probability of event $\mathcal{E}$ occurring \\
$\Gamma(.,)$. & Upper incomplete Gamma function \\
$\gamma(.,)$. & Lower incomplete Gamma function \\
$\mathbb{R}^{+}$ & The set of positive real numbers (including zero) \\
$\mathbb{R}^{d}$ & Euclidean space of dimension $d$ \\
$\mathbf{1}_{\{.\}}$ & Indicator function that takes value 1, when the enclosed argument \\
& $\{$.$\} holds; otherwise it takes value 0$ \\
\hline
\end{tabular}




\section{Frequently Used Symbols}

\begin{tabular}{|c|c|}
\hline Symbol & Meaning \\
\hline$S_{\imath}$ & Desired signal at the $i^{\text {th }} \mathrm{PR}$ of study \\
\hline$\gamma_{\imath}$ & $\begin{array}{l}\text { Exponentially distributed variable representing Rayleigh fading at } \\
\text { the desired signal link of the } i^{\text {th }} \mathrm{PR}\end{array}$ \\
\hline$\rho_{l, \mathrm{r}}$ & Mean received power, i.e., $\mathbb{E}\left(S_{\imath}\right)$ at the $i^{\text {th }} \mathrm{PR}$ \\
\hline$\varepsilon_{\imath}$ & Initial outage at the $i^{\text {th }} \mathrm{PR}$ of study \\
\hline$\varepsilon_{\imath, \mathrm{T}}, \varepsilon_{\imath, \mathrm{T}}\left(d_{\imath}\right)$ & Total outage at the $i^{\text {th }} \mathrm{PR}$ of study \\
\hline$\alpha$ & Path-loss exponent \\
\hline $\mathbb{J}$ & Initial unwanted signal set at the $i^{\text {th }} \mathrm{PR}$ of study \\
\hline $\mathbb{K}$ & New unwanted signal set at the $i^{\text {th }}$ PR of study \\
\hline$J$ & Net unwanted signal from set $\mathbb{I}$ \\
\hline$K$ & Net unwanted signal from set $\mathbb{K}$ \\
\hline$\Delta \varepsilon_{\imath}$ & $\begin{array}{l}\text { Additional outage at the } i^{\text {th }} \mathrm{PR} \text { of study due to new unwanted } \\
\text { signal set (or, interference) }\end{array}$ \\
\hline$\Delta \varepsilon_{\imath, \text { th }}$ & Additional outage constraint at the $i^{\text {th }} \mathrm{PR}$ of study \\
\hline$\lambda$ & Density of STs \\
\hline$\rho_{\mathrm{t}}(r, \theta), \rho_{\mathrm{t}}$ & Transmit power of the ST \\
\hline$\Delta \varepsilon_{\imath, \text { th }}$ & Additional outage constraint by the $i^{\text {th }} \mathrm{PR}$ of study \\
\hline$\eta_{\imath}$ & Background noise at the $i^{\text {th }} \mathrm{PR}$ of study \\
\hline$r_{1}$ & Inner radius or exclusion region of secondary network field-size \\
\hline$r_{2}$ & Secondary network field depth or outer radius \\
\hline$I_{\imath, \mathrm{s}}$ & Interference from the $\mathrm{SN}$ at the $i^{\text {th }} \mathrm{PR}$ of study \\
\hline$I_{\imath, \mathrm{p}}$ & Interference from the PN at the $i^{\text {th }} \mathrm{PR}$ of study \\
\hline$\Pi_{\imath, \mathrm{s}}$ & $\begin{array}{l}\text { Point process representing locations of the STs, conditioned on the } \\
i^{\text {th }} \text { PR of study }\end{array}$ \\
\hline$\Pi_{\imath, \mathbf{p}}$ & $\begin{array}{l}\text { Point process representing locations of the PTs, conditioned on the } \\
i^{\text {th }} \text { PR of study }\end{array}$ \\
\hline$\delta \varepsilon_{\imath, \mathbf{T}}$ & $\begin{array}{l}\text { Percentage increase in the total outage when the unbounded path- } \\
\text { loss model is chosen over the bounded path-loss model }\end{array}$ \\
\hline$Y_{k, 2}$ & Location of the $k^{\text {th }} \mathrm{PR}$ w.r.t to the $i^{\text {th }} \mathrm{PR}$ of study \\
\hline$X_{\jmath, 2}$ & Location of the $j^{\text {th }} \mathrm{ST}$ w.r.t to the $i^{\text {th }} \mathrm{PR}$ of study \\
\hline
\end{tabular}




\begin{tabular}{|c|c|}
\hline$\Gamma_{3,2}$ & $\begin{array}{l}\text { Exponentially distributed variable representing Rayleigh fading on } \\
\text { the interference link between the ST and the } i^{\text {th }} \mathrm{PR} \text { of study }\end{array}$ \\
\hline$\Gamma_{\jmath, k, \imath}^{\prime}$ & $\begin{array}{l}\text { Exponentially distributed variable representing Rayleigh fading on } \\
\text { the sensing channel between the } j^{\text {th }} \mathrm{ST} \text { and the } k^{\text {th }} \mathrm{PR} \text { under the } \\
\text { given location of the } i^{\text {th }} \mathrm{PR} \text { of study }\end{array}$ \\
\hline$\rho_{\mathrm{b}}$ & Probe power transmitted by the PRs on the sensing channel \\
\hline$\beta_{\imath, \text { th }}$ & $\begin{array}{l}\text { Decision threshold for ST to decide whether to transmit or not, } \\
\text { w.r.t the } i^{\text {th }} \text { PR of study }\end{array}$ \\
\hline $\mathbb{S}_{k, \imath}$ & $\begin{array}{l}\text { Set of deactivated STs that are not allowed to transmit around the } \\
k^{\text {th }} \mathrm{PR} \text { (at location } Y_{k, \imath} \text { ) conditioned on the } i^{\text {th }} \mathrm{PR} \text { of study }\end{array}$ \\
\hline$\Pi_{\imath, \mathrm{s}, \mathrm{t}}$ & $\begin{array}{l}\text { Point process representing the active STs that are allowed to trans- } \\
\text { mit conditioned on the } i^{\text {th }} \mathrm{PR} \text { of study }\end{array}$ \\
\hline$\Pi_{\imath, \mathrm{s}, \mathrm{t}}^{\prime}$ & $\begin{array}{l}\text { Point process representing the STs that are left by deactivating STs } \\
\text { around } i^{\text {th }} \text { PR only, conditioned on the } i^{\text {th }} \text { PR of study }\end{array}$ \\
\hline$\lambda_{\imath, \mathrm{s}, \mathrm{t}}^{\prime}(r)$ & Density of $\Pi_{\imath, \mathrm{s}, \mathrm{t}}^{\prime}$ at $(r, \theta)$, conditioned on the $i^{\text {th }} \mathrm{PR}$ of study \\
\hline$\varepsilon_{\imath, \text { th }}$ & Total outage constraint at the $i^{\text {th }}$ PR of study \\
\hline$\beta_{\imath, \text { th }}^{\text {opt }}$ & $\begin{array}{l}\text { Optimum decision threshold for ST to decide whether to transmit } \\
\text { or not, w.r.t the } i^{\text {th }} \mathrm{PR} \text { of study }\end{array}$ \\
\hline$N_{1}, N_{2}$ & $\begin{array}{l}\text { Number of active STs in the case of presumably exact exclusion re- } \\
\text { gion (formed considering no fading on the sensing channel) and the } \\
\text { soft exclusion region (formed by considering fading on the sensing } \\
\text { channel), respectively }\end{array}$ \\
\hline
\end{tabular}




\section{Chapter 1}

\section{Introduction}

\section{$1.1 \quad$ Motivation}

In the future, a plethora of devices (or networks) are expected to share a common spectrum. To meet the ever increasing applications requirements, bandwidth, which is limited, must be allocated to different networks in such a way that it is used in the most efficient manner. Spectrum measurements conducted at different places have revealed the spectrum utilization to vary significantly in time and space $[1,2]$. Accordingly, various spectrum sharing scenarios have been proposed in the literature, where two or more networks share the spectrum. A typical scenario is one where a primary network (PN) and secondary network (SN) share the spectrum. These SNs can be a set of femtocells or an ad hoc network in a cellular setup, or any other combination of heterogeneous networks. Other than PN-SN concept, even in case of unlicensed bands, networks like WiFi have shown tremendous growth, owing to inexpensive and easily deployable equipment.

Spectrum sharing networks are often overlaid or made to lie close to each other which causes significant interference to each other. The focus has been on the study of the effect of one network on the performance of other network. The principal performance metric in such studies at the physical layer is the outage suffered by the victim 
receiver of the incumbent network due to the new network. The network operators would like to get insights into the outage that a victim receiver node belonging to the PN would suffer from the deployment of a SN. Conversely, there is an opportunity for the new or SN to share the spectrum if it satisfies the outage constraint specified at the PN. It is also important to know what spectrum sharing gains can be achieved under the given outage constraint.

\subsection{Background}

There are primarily two spectrum sharing schemes that the SN can utilize to share the spectrum of the PN [3]. In one of these schemes, known as the underlay scheme, the SN could concurrently access the band of the primary user (PU) during the presence of the PU provided that the SN activities do not degrade the PU services beyond a certain level. In another scheme, known as the overlay scheme, secondary user (SU) could use the spectrum only during the absence of the PU. The latter scheme usually requires spectrum sensing and cognitive radio functionalities. However, adding these functionalities may increase the complexity of the secondary nodes to a level that can be economically infeasible, especially for large deployments. Moreover, the overlay scheme is also prone to sensing errors due to channel impairments or hardware inaccuracies that lead to the presence of some undesired active SUs [4].

Most of the previous works focused on the scenarios where the PU spectrum is accessed by a single SU, as given in the references in [3]. However, the networks consisting of large number of nodes, are characterized by the uncertainty in the number and the locations of the co-channel network nodes. The number of possible network configurations increases dramatically with the increase in the number of nodes. Hence, a deterministic approach used for few nodes is highly challenging to be scaled to large networks (as in femtocells and ad hoc networks) where the number as well as 
the positions of the nodes are random. Here, a mathematical tool known as stochastic geometry comes to the rescue for the study of the stochastic behaviour of the network [5-8]. In the study of multiple nodes, stochastic geometry is a promising tool which has shown its importance and usefulness in a number of fields including chemistry as well as forestry. It deals with the various spatial distributions of geometric objects. One of the well-known geometric models used in modeling the network nodes is the spatial point process. Owing to its complete randomness and analytical tractability, the most common spatial point process is the Poisson point process (PPP) or the homogeneous Poisson point process (HPPP). It is also the most basic point process from which other kinds of point processes can be derived by applying suitable operations.

The outage performance at any receiver, which depends on the interference received at this victim receiver, requires the characterization of the interference coming from the randomly distributed interferers [9]. For spectrum sharing networks, these interferers are the secondary transmitters (STs) in the SN as well as the primary transmitters (PTs) in the PN. In a parallel line of works for single networks like CDMA, interferers are co-channel transmitters in the same network. Both the cases, namely the spectrum sharing networks and the single networks, share some general results derived for the set of interferers that are distributed as a point process around the receiver of study. An invited paper [9] on the network interference and its application provides a comprehensive presentation of the state of art in this area. The HPPP was first considered in [10] for the single network where the interfering transmitters are deployed as an HPPP around the victim receiver. In that case, for the interferers are deployed on a 2-D plane and a path-loss exponent of $\alpha=4$, the authors obtained the interference distribution in a closed-form. For other cases, the closed-form for the interference distribution does not exist. Hence, the common approach followed is to perform a distribution fit for the aggregate interference through the moments or 
cumulants (e.g., mean and variance) matching approach [11-14].

In the early works, generally a typical receiver is taken and the interferers are assumed to be randomly distributed around that receiver according to an HPPP. Sometimes, a presumably circular exclusion region or a guard zone is assumed around the receiver to alleviate the profound effect of the nearby interfering transmissions. The new HPPP for the interferers is formed outside the exclusion region by thinning or removal of the points of the original HPPP inside the exclusion region. In case of spectrum sharing, [11] studied the outage probability at the typical PR by doing the lognormal distribution fit for the interference coming from the SN (or, STs). In [12], the authors approximated the aggregate interference coming from the SN with the shifted lognormal distribution and studied the outage at the typical PR. They also incorporated the distance dependent distributed sensing mechanism for STs to decide whether to transmit or not. In case of TV bands, [15] proposed a set up where the PRs with guard zones around them are distributed randomly inside a large presumably exact exclusion region centered around a PT. The STs are randomly distributed outside the PT exclusion region. The authors found the relationship between the derived outage bound at the $\mathrm{PR}$ and the exclusion regions around the PR and PT in the worst case scenario, i.e., when the PR is at the edge of the exclusion region. Similarly, in an another work on TV bands [16], the authors showed that the lognormal or shifted lognormal approximations lead to under or over estimation of outages under large channel variations. They proposed the exclusion region around the PR, conservative than both of the approximations, to satisfy the outage constraint at the PR of study.

Furthermore, the validation of Gaussian distribution fit for the interference with respect to the field-size (area over which interferers are distributed) and the exclusion region is examined in [17] using the cumulants matching approach. Another approach taken in [18] to study the outage-density tradeoffs in the low outage regions is to 
consider the dominating ST only rather than all the STs. In the presence of Rayleigh fading, the outage can be expressed in terms of a Laplace transform of the interference distribution [19]. In [20], the authors derived the outage experienced by the PR in a closed-form in the Rayleigh fading environment, with the STs distributed over an infinite area.

One parameter that has not drawn attention in the earlier works is the field-size of the interferers. Only recently, [21] studied the impact of the SN field-size and the exclusion region on the spectrum sharing opportunities by doing the interference distribution fit through the cumulants matching approach. Furthermore, in [17], the dominant region (the region mainly contributing to the aggregate interference) impacting the spectrum sharing opportunities is addressed. In [22], the authors obtained the general expressions for the moments of the interference coming from the finite field of interferers.

In case of a single network, identical TX-RX pairs are considered where the interferers are the co-channel transmitters in the same network. Authors in [19] derived numerically integrable expressions for the outage at any receiver of the single-hop ad hoc network when the interfering co-channel transmitters are distributed over an infinite area. Similarly, authors in [23] studied a single ad hoc network by assuming exclusion regions around all the receivers. Since every receiver is statistically identical, the outage experienced by any typical receiver reflects the outage of the network. The transmitters left after the thinning process no longer form a PPP, however, this work approximated it by HPPP. Through simulations, they showed that for large exclusion regions, aggregate interference distribution can be approximately fitted to the Gaussian distribution. A similar observation is made in [13] for a CDMA network to study the outage. With Rayleigh fading, [24] obtained the outage using numerical techniques for infinite field size of the interferers.

In the literature on spectrum sharing overlaid networks, generally both network 
nodes are assumed to be distributed as HPPP with identical primary receiver-primary transmitter (PR-PT) and secondary receiver-secondary transmitter (SR-ST) pairs. Outage at any typical PR, which is same for all PRs, is studied since every PR experiences same statistics of aggregate interference from the SN and PN. The case of femtocells sharing the spectrum of the cellular network in the uplink case has been considered in [25] where both the femtocell BSs and the macrocell (cellular) users are distributed according to HPPPs. They assumed scaled Gaussian distribution for the interference coming from out of the cell to derive the macro-cell (or, basestation) outage probability. Similarly, uplink case of ad hoc network overlaid on the cellular network is considered and the outage bound at the cellular BS is obtained in [26]. In a related line of work for evaluating the transmission capacity [27] of the overlaid networks, [28] derived the outage suffered by the PN in the Rayleigh fading by assuming both network nodes to be distributed according to HPPP for asymptotically small outage.

\subsection{Mathematical Preliminaries}

In this section, we provide some mathematical preliminaries for point processes that are used in the analysis and discussions presented in this thesis.

Point processes are useful as statistical models in the analysis of the pattern of points, where the points represent the positions of the objects of study. As an application of the point process in the study of wireless networks, these points represent the nodes in the wireless network in the Euclidean space $\mathbb{R}^{d}$ (generally, $d=2$ is

considered) $[5,6]$. Point process theory allows to study the average behavior of a network over many spatial realizations, where the nodes are placed according to some probability distribution. Owing to its purely random behaviour, an important and the popular class of spatial point processes is the Poisson point process. 


\subsubsection{Poisson Point Process}

A Poisson point process $\Pi$ on $S \subseteq \mathbb{R}^{d}$ with mean measure $\mu(S)$ is a point process possessing the two following properties $[7,8,29]$ :

- The number of points in $S$ follows a Poisson distribution with mean $\mu(S)$, i.e.,

$$
\mathcal{P}(N(S)=k)=\frac{\mu(S)^{k} e^{-\mu(S)}}{k !} .
$$

- The numbers of points of $\Pi$ in $\mathrm{k}$ disjoint subsets $S_{1}, S_{2}, \ldots, S_{k}$ form $k$ independent random variables of Poisson distribution, for arbitrary $k$.

\subsubsection{Laplace Functional $[7,8,29]$}

In order to perform the outage analysis, we need to study the aggregate interference power which is the sum of the interferences coming from all the interferers. Each interference component is a function of the randomly distributed interfering node.

Laplace functional (Laplace transform of the distribution of a function) $\sum_{X \in \Pi} f(X)$ of any general point process can be written as

$$
\mathcal{L}_{\Pi}(f) \triangleq \mathbb{E}\left[e^{-\int_{S} f(x) \Pi(\mathrm{d} x)}\right]=\mathbb{E}\left[e^{-\sum_{X \in \Pi} f(X)}\right]
$$

where $f$ is a non-negative function on $\mathbb{R}^{d}$. In case, $\Pi$ is a Poisson process, then according to the Campbell theorem, the Laplace functional is given by

$$
\mathcal{L}_{\Pi}(f)=\exp \left\{\int_{S}\left(e^{-f(x)}-1\right) \mu(\mathrm{d} x)\right\} .
$$


The above condition holds if

$$
\int_{S} \min (|f(x)|, 1) \mu(\mathrm{d} x)<\infty .
$$

\subsubsection{Marked Poisson Point Process $[8,29]$}

Now, in order to associate a characteristic with a point $X \in \Pi$, a random variable $m_{X}$, known as the mark of $X$, is defined taking values in some space $M$. The pair $\left(X, m_{X}\right)$ is known as the the random point $X^{\prime}$ of the point process $\Pi^{\prime}$ in the product space $S \times M ; S \subseteq \mathbb{R}^{d}$. Hence, we can write

$$
\Pi^{\prime}=\left\{\left(X, m_{X}\right) ; X \in \Pi\right\}
$$

Now, if $\Pi$ is a Poisson process with mean $\mu(S)$, then according to the Marking Theorem, $\Pi^{\prime}$ is a Poisson process provided that the distribution of $m_{X}$ does not depend on the other points of $\Pi$, and that the $m_{X}$ for different $X$ are independent. Accordingly, the Campbell theorem is revised as

$$
\mathcal{L}_{\mathrm{II}^{\prime}}(f)=\exp \left\{\int_{S \times M}\left(e^{-f(x, m)}-1\right) \mu(\mathrm{d} x) p(x, \mathrm{~d} m)\right\},
$$

where $f\left(X, m_{X}\right)$ is any measurable function on $S \times M$ and $p(x,$.$) is a probability$ distribution on $M$ depending on $x \in S$.

\subsection{Thesis Contribution}

Despite recent advancements in the application of stochastic geometry to model the wireless networks, analytical studies are tractable only for few point processes, mainly PPP [6]. These point process models can accommodate uncoordinated type SN; 
e.g., ad hoc network, to some extent. However, such an assumption for incumbent networks like the cellular network might not correspond to reality, where the nodes are coordinated and there is an inherent separation between the nodes. Given the complex nature of the interaction between the PN nodes, the outage analysis at a $\mathrm{PR}$ of a PN that shares spectrum with a $\mathrm{SN}$ is highly challenging. As a result, in the previous works, either the interference from the incumbent network is not considered, or its PTs are assumed to be distributed independently and identically. Moreover, the interferers (STs or PTs or both) are assumed to be distributed over an infinite region for analytical simplicity. Such asymptotic studies give pessimistic results, and therefore, can lead to missing the spectrum sharing opportunities. Additionally, in the previous works, a presumably exact exclusion region has been assumed around the node (or, nodes) in the fading environment. However, it is impossible to define a physical boundary of the exclusion region, since the exclusion region is formed through signaling between the nodes and the signaling channels are bound to be affected by fading. Hence, there is a need for mathematical expressions that provide insights into the analytical aspect of such problems while maintaining low complexity. The contributions of this work are as follows:

- We derive an expression (2.11) for the additional outage caused by a new independent set of unwanted signals, which may comprise of interference or noise or both, assuming the well-accepted model of Rayleigh fading on the desired signal link. The result does not require the distribution of the initial unwanted signal set provided that the outage due to this distribution is known. As a corollary of this, it turns out that the additional outage caused by a new unwanted signal set is a decreasing function of the initial outage.

- The derived equation is applied in the context of spectrum sharing to find the additional outage at the PR due to the deployment of a SN. It does not 
need the knowledge of the distribution of the interference coming from the PN provided outage in the absence of SN is known. A closed-form expression for the outage at the victim receiver due to an annular sector region (which may include a presumably exact exclusion region, and a finite or infinite outer radius) of interferers is derived. Conversely, under a given outage constraint, spectrum sharing opportunities are analyzed with respect to the field size and are shown to be significantly dependent on the exclusion region. Conditions are obtained for which an infinite field size can lead to the loss of spectrum sharing opportunity. Furthermore, the spectrum sharing gains (maximum average number of STs) are derived for the SN for different spatial deployments. We also compare the singular and non-singular path-loss models under the given framework. Our results indicate that, for a given additional outage constraint, the spectrum sharing gains are higher for those networks that already experience a higher initial outage (which in turn depends on the type of PN). It follows that the interfering PTs should not be ignored in the spectrum sharing analysis.

- As an application, we extend the outage analysis for the single PR to the randomly but not necessarily independently distributed cellular PRs. We discuss the spectrum sharing scenario where the cellular network shares its spectrum with an ad hoc network in the uplink case. We make use of the sensing mechanism rather than the presumably exact exclusion region to decrease the profound effect of the nearby STs, and accordingly, an upper bound for the outage experienced by the PRs is derived. This bound is tight where the co-channel PRs are spatially separated by relatively significant distances, which is the case in cellular networks. It is well known that the sensing mechanism is quite significant in decreasing the outage suffered by the PRs. We provided this effect of sensing mechanism in a closed form expression. For the maximum spectrum 
sharing gains, we optimize the decision threshold (to decide on whether to transmit or not) used by the STs under the given outage constraints. Additionally, in our study, every co-channel primary signal link may have different system parameters. Furthermore, we observe that the soft exclusion region formed by considering fading on the sensing channel is better than the presumably exact exclusion region (with no fading on the sensing channel) in achieving higher spectrum sharing gains.

\subsection{Thesis Structure}

The rest of this thesis is organized into three Chapters as follows. Chapter 2 presents a general result for the outage that a receiver experiences from two independent unwanted signal sets. Along with the state of art, the significance of the derived result is shown in the context of spectrum sharing in Chapters 3 and 4 . The outage at a victim $\mathrm{PR}$ is analyzed in terms of various system parameters, specifically, the field size of the secondary network in Chapter 3. Then, in Chapter 4, the case of overlaid cellular and ad hoc networks is presented, incorporating the sensing mechanism. Finally, we present the conclusions and suggest future work items in the last chapter.

\subsection{Manuscripts Accepted, Submitted, and In Progress}

In this section, a list of the manuscripts accepted, submitted or in-progress based on the work in this thesis is presented.

- A. Kahlon, S. Szyszkowicz, S. Periyalwar, and H. Yanikomeroglu, "Identification of spectrum sharing opportunities for a finite field secondary network through an exact outage expression under Rayleigh fading," accepted to IEEE Personal Indoor Mobile, Radio Communications, 2011. This manuscript contains results from Chapter 3. 
- A. Kahlon, S. Szyszkowicz, S. Periyalwar, and H. Yanikomeroglu, "Analysis of additional outage in spectrum sharing with Rayleigh-faded signal link," under review in IEEE Transactıons on Wireless Communıcatıons. This manuscript contains results from Chapters 2 and 3.

- A. Kahlon, S. Periyalwar, and H. Yanikomeroglu, "Outage in a cellular network overlaid with an ad hoc network: the uplink case," accepted to IEEE Personal Indoor Mobıle, Radıo Communıcatıons 2011. This manuscript contains some of the results from Chapter 4.

- A. Kahlon, S. Periyalwar, and H. Yanikomeroglu, "Outage in a cellular network overlaid with an ad hoc network" under preparation for journal submission. This manuscript is to contain and extend the results from Chapter 4. 


\section{Chapter 2}

\section{Outage due to Independent Unwanted Signals}

\section{$2.1 \quad$ Introduction}

In order to analyze the outage at a receiver node, the total unwanted signal at that node needs to be studied. This unwanted signal can be the interference or noise or both. We derive a fundamental equation (2.12) for the outage caused by two independent unwanted signal sets, assuming that the useful signal at the receiver experiences Rayleigh fading. The expression does not depend on the distribution of the net unwanted signal from one of these sets; it only depends on the outage due this unwanted signal set. Section 2.2 gives a general system model of the problem we intend to solve, and gives the starting point for its analysis. In Section 2.3, we give the main results for the outage due to two independent unwanted signal sets. The result is significant where the distribution of the net unwanted signal from one of the unwanted signal set is not known or hard to characterize. Furthermore, an interesting observation is made from the derived results that the additional outage due to a new unwanted signal set will be lesser for the user which experiences higher initial outage (outage in the absence of new unwanted signal set). This observation does not require the knowledge of the distribution of any of the net unwanted signals from the two unwanted signal sets. Additionally, as an another corollary, the derived 
result is extended to any number of unwanted signal sets where the outages due to these sets only are known.

\subsection{Two Independent Unwanted Signals}

We begin by formulating a very general problem, where the two independent sets of signals, i.e., $\mathbb{J}$ and $\mathbb{K}$ are unwanted (or, interfering) at the victim receiver. These sets of unwanted signals can be from the same source, or can be from two different sources where the statistical layouts of the sources may take any form. These unwanted signal sets can consist of noise or interference or both. We only require two conditions: that the two sets of unwanted signals be independent of each other, and that the desired signal at the victim receiver be Rayleigh distributed.

Let the desired signal power received at the victim receiver be

$$
S_{1}=\gamma_{1} \rho_{1, r}
$$

where $\gamma_{1}$ is an exponentially distributed variable with unit mean representing Rayleigh fading and $\rho_{1, r}$ is the mean received power. Suppose, the outage probability at the victim receiver due to the net unwanted signal $J$ from the set $\mathbb{J}$ be $\varepsilon_{1}$, given by

$$
\mathcal{P}\left(\frac{S_{1}}{J}<\beta_{1}\right)=\varepsilon_{1}
$$

where $\beta_{1}$ is the SIR or SNR or SINR threshold depending on the set of unwanted signal. Now, in the presence of both $J$ and $K$, where $K$ is net unwanted signal from $\mathbb{K}$, the total unwanted signal at the victim receiver node is given by $J+K$. The addition of unwanted signals in power is a common assumption $[9,19,30]$. The outage 
due to both unwanted signals can be expressed as

$$
\mathcal{P}\left(\frac{S_{1}}{J+K}<\beta_{1}\right)=\varepsilon_{1}+\Delta \varepsilon_{1}
$$

where $\Delta \varepsilon_{1}$ is the additional outage in the presence of $\mathbb{K}$. This is the most general form of the problem that we intend to solve.

\subsection{Outage Analysis}

We analyze the outage at the victim node for the general case of independent unwanted signal sets, i.e., $\mathbb{J}$ and $\mathbb{K}$. Consider the following main result derived in the form of theorem and its proof:

Theorem 1. Outage $\varepsilon_{1, \mathrm{~T}}$ at the recevver due to the independent unwanted signal sets $\mathbb{J}$ and $\mathbb{K}$, assuming the desired signal power us exponentıally distributed with mean $\rho_{\imath, r}$, is given by

$$
\varepsilon_{1, \mathrm{~T}}=1-\left(1-\varepsilon_{1}\right) \mathbb{E}\left(e^{-\kappa_{1} K}\right)
$$

where $\kappa_{1}=\frac{\beta_{1}}{\rho_{1, r}}, \beta_{1}$ is the threshold $S N R$ or SIR or SINR ratıo, $K$ us the net unwanted signal from the set $\mathbb{K}$ and $\varepsilon_{1}$ is the outage due to $\mathbb{J}$ only.

Proof. In the presence of $\mathbb{J}$ only, using (2.1) and (2.2), the outage expression is given as

$$
\mathcal{P}\left(\frac{\gamma_{1} \rho_{1, r}}{J}<\beta_{1}\right)=\varepsilon_{1}
$$

Substituting $\kappa_{1}=\frac{\beta_{1}}{\rho_{1, r}}$ in the above equation, we can write

$$
\begin{aligned}
& \mathbb{E}\left(\mathcal{P}\left(\gamma_{1}<\kappa_{1} J \mid J\right)\right)=\varepsilon_{1} \\
\Rightarrow & \mathbb{E}\left(e^{-\kappa_{1} J}\right)=1-\varepsilon_{1} .
\end{aligned}
$$

Now in the presence of both $\mathbb{J}$ and $\mathbb{K}$, and proceeding in the same manner as above, 
from (2.3), we can write

$$
\mathbb{E}\left(e^{-\kappa_{1}(J+K)}\right)=1-\left(\varepsilon_{1}+\Delta \varepsilon_{1}\right) .
$$

Since both the unwanted signal sets $\mathbb{I}$ and $\mathbb{K}$ are independent, we replace $\mathbb{E}\left(e^{-\kappa_{1}(J+K)}\right)=$ $\mathbb{E}\left(e^{-\kappa_{1} J}\right) \mathbb{E}\left(e^{-\kappa_{1} K}\right)$. In the above equation:

$$
\mathbb{E}\left(e^{-\kappa_{1} J}\right) \mathbb{E}\left(e^{-\kappa_{1} K}\right)=1-\left(\varepsilon_{1}+\Delta \varepsilon_{1}\right) .
$$

From (2.6) and (2.8), we obtain

$$
\mathbb{E}\left(e^{-\kappa_{1} K}\right)=1-\frac{\Delta \varepsilon_{1}}{1-\varepsilon_{1}} .
$$

Or,

$$
\varepsilon_{1, \mathrm{~T}}=\varepsilon_{1}+\Delta \varepsilon_{1}=1-\left(1-\epsilon_{1}\right) \mathbb{E}\left(e^{-\kappa_{1} K}\right) .
$$

We can write the above result in the form of a Laplace transform:

$$
\mathcal{L}_{K}\left(\kappa_{1}\right)=1-\frac{\Delta \varepsilon_{1}}{1-\varepsilon_{1}}
$$

Also,

$$
\varepsilon_{1, \mathrm{~T}}=\varepsilon_{1}+\Delta \varepsilon_{1}=1-\left(1-\varepsilon_{1}\right) \mathcal{L}_{K}\left(\kappa_{1}\right)
$$

\subsection{Significance of the Derived Result}

The above equations, i.e, (2.11) and (2.12), show that we only need the initial outage condition $\varepsilon_{1}$ due to $J$ at the victim receiver, rather than the entire distribution of $J$, to study the additional outage $\Delta \varepsilon_{1}$ (or, the total outage $\varepsilon_{1, \mathrm{~T}}$ ), which results in a 
significant simplification in the analysis. Initial outage can be easily found through the usage statistics on whether the user is in the outage or not (or, other empirical methods), as compared to the tough task of measuring and characterizing the unwanted signal $J$. Sometimes, there might be a situation when the unwanted signal set at the receiver comprises of two independent signal sets $\mathbb{J}$ and $\mathbb{K}$, and it is not feasible to find the probability distribution of the net unwanted signal $J$ from one of the signal set J. In that case, the equation (2.12) can be useful in obtaining the total outage due to $J+K$ without the need of characterizing $J$, provided that desired signal link is Rayleigh faded and the outage due to $J$ is known. Alternatively, we can obtain outage due to any unwanted signal with unknown distribution that can be decomposed into two independent sets of unwanted signals, when the distribution of the net unwanted signal from one of these sets and outage due to the other unwanted signal set are known.

Furthermore, we make an interesting observation from equation (2.11), without the need of the knowledge of the distributions of any of the unwanted signals from the two unwanted signal sets, as given below.

Corollary 1. The additional outage $\Delta \varepsilon_{1}$ is a decreasing function of the initial outage $\varepsilon_{1}$ for some $K$ and $\kappa$.

Hence, if two identical users experience the same new interference (or, noise) and different initial unwanted signal sets, additional outage will be higher for that user which is experiencing lesser initial outage.

Corollary 2. The total outage $\varepsilon_{\mathrm{T}}$ due to $N$ independent unwanted signal sets is given by

$$
\varepsilon_{\mathbf{T}}=1-\prod_{\imath=1}^{N}\left(1-\varepsilon_{\imath}\right),
$$

where $\varepsilon_{\imath}$ is the outage due to $\imath^{\text {th }}$ unwanted signal set only. 
This is more general form of the theorem derived where in order to obtain the total outage, only the outages caused by the constituent unwanted signal sets are required; not their whole distributions.

\subsection{Summary}

In this chapter, we give the fundamental outage equation for a general case where a receiver experiences outage due to two independent unwanted signal sets. These sets can be from the same source or different sources. Examples of such sources can be set of interferers in the same network or different network. The outage at the victim receiver due to two independent unwanted signal sets is derived in (2.12), under the given outage due to one of these sets, but without the need of characterizing the

unwanted signal from the other set. As a corollary, additional outage due to a new unwanted signal set is found to be a decreasing function of the initial outage (outage in the absence of the new unwanted signal set). This observation does not require the knowledge of the distribution of the net unwanted signals from either of the unwanted signal sets. The derived result is extended to any number of unwanted signal sets where the outage caused by these sets are known. This is given in the second corollary. The derived result is significant when the distribution of the net unwanted signal from one of the unwanted signal set is hard to characterize. In the following chapters, the application of this derived equation in the spectrum sharing scenario is shown. 


\section{Chapter 3}

\section{Spectrum Sharing for Finite Field Secondary}

\section{Network}

\subsection{Introduction}

In this chapter, an application of the equation (2.11) derived in the previous chapter is shown in a spectrum sharing scenario based on the assumption that there is some additional outage tolerance available in the PU. The SN share the spectrum of the PU in the underlay scheme [31], under the condition that the PU services should not be affected beyond a certain threshold set by the PU. Without actually deploying the SN, the network operators would like to get insights into the additional outage experienced by the PR with the deployment of SN. Conversely, there is an opportunity for the SN to share the spectrum if it satisfies the additional outage constraint specified by the PR. Or, in other words, SN parameters should be designed in such a way that the outage condition at the PR is satisfied. The PN would normally have knowledge of the initial outage that the PR already experiences, which can be obtained from the usage statistics. It is also important to know what spectrum sharing gains (notably, the maximum number of STs) can be achieved given the additional outage constraint.

In order to analyze the outage at a PR, the combined effect of the total interference and noise received at that receiver needs to be studied. This total interference is a 
combination of the interference from the $\mathrm{SN}$ (SN interference), as well as from the other sources of interference, especially the PR's own PN (PN interference). However, in the previous works, due to the potentially complex nature of the interaction among the PN's nodes, either the PN interference is not considered, or the co-channel PTs are assumed to be distributed as the points of an HPPP [29] over an infinite area. Since most of the PNs are already interference-limited, and therefore the PN interference is not negligible, ignoring it might not correspond to reality. As for the distribution of the network nodes, SN networks might reasonably be modeled as points of an HPPP to get the insights, since they are mostly opportunistic networks such as ad hoc networks (or any other kind of uncoordinated network). However, in the PNs, which are mainly licensed networks such as legacy cellular, the interfering PTs may be expected to interact among each other in complex ways that an HPPP model cannot represent accurately.

A significant amount of literature focuses on scenarios where only the STs are deployed around the PR under study. Sometimes, a presumably exact exclusion region (or, protection region) around this $\mathrm{PR}$ is assumed, to decrease the profound effect of nearby STs. Several works $[11,12,32-35]$ perform curve fitting of the SN interference distribution using the moments (or cumulants) matching approach or numerical methods. In some of these cases, only the asymptotically small outage conditions are studied. Another approach to study the trade-off between the ST density and outage in low outage regions is to consider only the dominant ST [18]. Outage bounds at the typical PR are obtained in [36] for TV band, with exclusion regions around both the PT and PR. Similarly, [16] obtained the approximation to the guard zone around the PR for the TV bands using cumulants. The maximum density of the STs is derived for Rayleigh fading channels in [20] under a given outage constraint for infinite secondary interferers field size [16].

The only works that include PN interference are those in the parallel line of 
research examining transmission capacity [27] for overlaid networks with statistically identical receivers. However, here either the PTs are distributed according to an HPPP over an infinite region $[25,26,37,38]$, or a distribution fit is done for the PN interference [25].

Moreover, in most works, STs are distributed over an infinite field for analytical simplicity. This approach is pessimistic in the sense that the SN interference is overestimated, and spectrum sharing opportunities are lost. The effect of the STs' field size is studied in $[21,39]$, where the conditions for an infinite field to be a valid approximation to a finite field are explored through outage bounds and cumulants matching respectively. Again, these works did not consider PN interference. Outside the context of spectrum sharing, integral expressions for the outage in a single ad hoc network are given in [24], with the interferers distributed over an annular region. A bound for the outage is derived in closed-form, but only for an infinite outer radius.

The contributions of this chapter are as follows. The equation derived in the previous chapter is applied in the context of spectrum sharing to find the additional outage at the typical PR of the PN due to the deployment of a SN. Conversely, we obtain the expression that the SN needs to satisfy in order to exist with the $\mathrm{PR}$, under a given outage constraint by the PR. We derive an expression for the additional outage when the STs are distributed as a general PPP over a given area. More specifically, when the STs are distributed as an HPPP, we give an expression for the maximum density of the SN for a given additional outage constraint. We find this expression in a closed-form when the HPPP is distributed over an annular sector region (which may include an exclusion region, and a finite or infinite outer radius). We confirm this result through Monte Carlo simulations. Furthermore, the spectrum sharing opportunities are analyzed with respect to the field size and are shown to be significantly dependent on the exclusion region. Conditions are obtained for which an infinite field size can lead to the loss of spectrum sharing opportunity. Additionally, 
the spectrum sharing gains (maximum average number of STs) are derived for the SN for different spatial deployments. Furthermore, the effect of the ST transmit power on the outage is analyzed with respect to field-size. We also compare the singular and non-singular path-loss models with respect to the outage metric. Our results indicate that, for a given additional outage constraint, the spectrum sharing gains are higher for those PNs that already experience a higher initial outage (which in turn depends on the type of PN). It follows that the PN interference should not be ignored in spectrum sharing analysis. It is worth mentioning that the derived closed-form results are also applicable for any set of interferers in general, not only the STs.

\subsection{System Model}

Without the loss of generality, we begin by giving a system model of the spectrum sharing scenario where a typical PR at origin experiences additional outage from the $\mathrm{SN}$. Let the useful or desired signal received at the PR is given be

$$
S_{1}=\gamma_{1} \rho_{1, \mathrm{r}},
$$

where $\rho_{1, \mathrm{r}}$ is the mean received power, $\gamma_{1}$ is the exponentially distributed random variable representing Rayleigh fading on the desired signal link. In the absence of the $\mathrm{SN}$, let the unwanted signal power consists of interference $\left(I_{1, \mathrm{p}}\right)$ from the PN and the noise $\left(\eta_{1}\right)$. Let the initial outage probability at the PR due to the $I_{1, \mathrm{p}}+\eta_{1}$ be $\varepsilon_{1}$, given by

$$
\mathcal{P}\left(\frac{S_{1}}{I_{1, \mathrm{p}}+\eta_{1}}<\beta_{1}\right)=\varepsilon_{1},
$$

where $\beta_{1}$ is the SINR threshold. Now in the presence of SN, the net unwanted signal at the PR is given by $I_{1, \mathrm{p}}+I_{1, \mathrm{~s}}+\eta_{1}$, where $I_{1, s}$ is the SN interference. In the presence 
of $\mathrm{SN}$, the additional outage is $\Delta \varepsilon_{1}$. The new outage expression is given by

$$
\mathcal{P}\left(\frac{S_{1}}{I_{1, \mathrm{p}}+I_{1, \mathrm{~s}}+\eta_{1}}<\beta_{1}\right)=\varepsilon_{1}+\Delta \varepsilon_{1}
$$

In order to incorporate the randomness in the location of the STs, we make use of the spatial point process theory [6-8]. Let the STs be distributed as the points of a point process $\Pi_{\mathrm{s}}=\left\{X_{\jmath}\right\}$ over $S \subseteq \mathbb{R}^{2}$. With respect to $\mathrm{PR}$ at origin, we define $\Pi_{1, \mathrm{~s}}=\left\{\left(X_{\jmath, 1}, \Gamma_{\jmath, 1}\right) \mid X_{\jmath} \in \Pi\right\}$ over the product space $S \times \mathbb{R}^{+}$, where $X_{\jmath, 1}=X_{\jmath}$ and $\Gamma_{\jmath, 1}$ is the mark associated with the ST at $X_{\jmath, 1}$, representing fading on the interfering channel between this ST and the typical PR. The marks are distributed identically and independently with distribution $f_{\Gamma}(y)$ for every $\jmath$, and independently of $X_{3}$.

In the case where $\Pi_{s}$ is a Poisson point process, then from the marking theorem [29], $\Pi_{1, \mathrm{~s}}$ is also a Poisson point process. We then apply Campbell's theorem [29] to $\Pi_{1, \mathrm{~s}}$, using (1.6), to obtain the Laplace transform of the distribution of $I_{1, s}$ at the origin after some mathematical manipulations:

$$
\mathcal{L}_{I_{1, s}}(x)=\exp \left\{\int_{S} \int_{0}^{\infty}\left(e^{-x y \rho_{\mathrm{t}}(r, \theta) g(r)}-1\right) \lambda(r, \theta) r f_{\Gamma}(y) \mathrm{d} y \mathrm{~d} r \mathrm{~d} \theta\right\}
$$

where $\lambda(r, \theta)$ is the density of STs in $\Pi_{\mathrm{s}}$ at $(r, \theta), \rho_{\mathrm{t}}(r, \theta)$ is the power transmitted by the ST at location $(r, \theta), \alpha$ is the path-loss exponent, $\Gamma$ is an exponentially distributed random variable with unit mean, and $g(r)$ is the large scale path-loss function. As done in previous works, $\alpha>2$ is taken when STs are distributed over an infinite region for Campbell's theorem to hold $[9,12]$.

- For an HPPP, we have $\lambda(r, \theta)=\lambda$.

- In the case that all the STs transmit at the same power, we have $\rho_{\mathrm{t}}(r, \theta)=\rho_{\mathrm{t}}$. 


\subsection{Outage at a PR}

In the case of spectrum sharing networks, we substitute $J=I_{1, \mathrm{p}}+\eta_{1}$ and $K=I_{1, \mathrm{~s}}$ in the derived equation (2.11) to obtain the additional outage at the PR due to the $\mathrm{SN}$. It avoids the highly challenging task of characterizing the complex nature of the sum of the PN interference and the noise $I_{1, \mathrm{p}}+\eta_{1}$, and gives an alternative way to get some insights into the spectrum sharing systems without unrealistic assumptions or asymptotic outage studies. Moreover, it is useful as well as feasible to find $\varepsilon_{1}$ from the usage statistics from the network operator, rather than taking interference measurements.

Now, we apply the derived equation (2.11) for the case of spectrum sharing between the PN and the SN by substituting in (2.11).

$$
\mathcal{L}_{I_{1, s}}\left(\kappa_{1}\right)=1-\frac{\Delta \varepsilon_{1}}{1-\varepsilon_{1}}
$$

where $\kappa_{1}=\frac{\beta_{1}}{\rho_{1, \mathrm{r}}}$. Given the initial outage $\varepsilon_{1}$ at the PR before the deployment of a $\mathrm{SN}$ and the maximum additional outage $\Delta \varepsilon_{1, \text { th }}$ that it can tolerate, the SN needs to satisfy

$$
\mathcal{L}_{I_{1, \mathrm{~s}}}\left(\kappa_{1}\right) \geq 1-\frac{\Delta \varepsilon_{1, \mathrm{th}}}{1-\varepsilon_{1}}
$$

at the PR. This equation also helps to design the parameters of the SN to satisfy this outage constraint $\Delta \varepsilon_{1, \text { th }}$ given by the PR.

This general result can be applied in some of the spectrum sharing scenarios of interest in current research.

\section{SN Distribution: General PPP}

We now perform the outage analysis for a typical PR when the STs are distributed as a PPP on $S \subseteq \mathbb{R}^{2}$ as shown in Fig. 3.1. We may rewrite (3.4) after some mathematical 


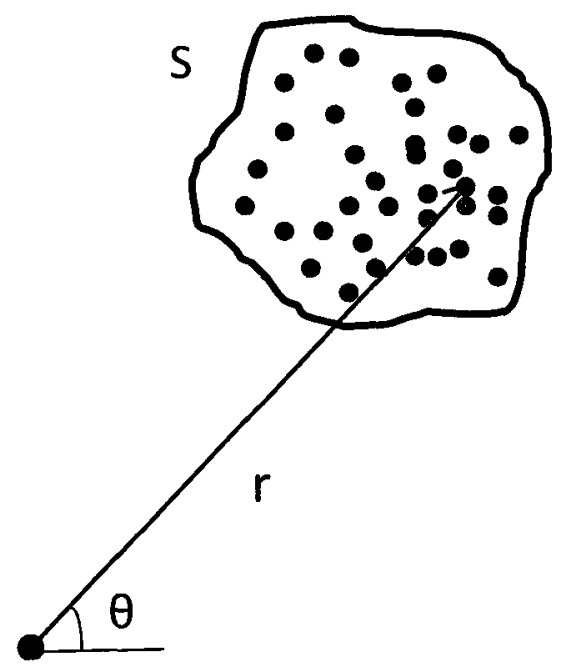

Figure 3.1: Set of STs distributed as a PPP over some general region.

manipulations, using $f_{\Gamma}(y)=e^{-y}$ for Rayleigh fading, to obtain

$$
\mathcal{L}_{I_{1, \mathrm{~s}}}(x)=\mathbb{E}\left(e^{-x I_{1, s}}\right)=\exp \left\{\int_{S}\left(\frac{-x \rho_{\mathrm{t}}(r, \theta)}{x \rho_{\mathrm{t}}(r, \theta)+r^{\alpha}}\right) \lambda(r, \theta) r \mathrm{~d} r \mathrm{~d} \theta\right\}
$$

The above equation depends on the region over which STs are distributed. With the help of numerical techniques, it can be solved and put in (3.5) .

\section{SN Distribution: HPPP over a General Region}

For constant density $\lambda(r, \theta)=\lambda$, we can write the above equation (3.7) as

$$
\mathcal{L}_{I_{1, \mathrm{~s}}}\left(\kappa_{1}\right)=\exp (-\lambda C)
$$

where

$$
C=\int_{S} \frac{\kappa_{1} \rho_{t}(r, \theta)}{\kappa_{1} \rho_{\mathrm{t}}(r, \theta)+r^{\alpha}} r \mathrm{~d} r \mathrm{~d} \theta
$$


depends on the SN parameters and $S$. Hence, the maximum density of STs $\lambda_{\max }$ under the given outage constraint $\Delta \varepsilon_{1, \text { th }}$ by the PR using (3.6) is given by

$$
\lambda_{\max }=-\frac{1}{C} \log \left(1-\frac{\Delta \varepsilon_{1, \mathrm{th}}}{1-\varepsilon_{1}}\right) .
$$

For a given $C$, the density of the STs $\lambda_{\max }$ represents the spectrum sharing gains under the given additional outage constraint.

Conversely, the additional outage suffered by the PR under the given density of STs is

$$
\Delta \varepsilon_{1}=\left(1-\varepsilon_{1}\right)(1-\exp (-\lambda C))
$$

Or, the total outage $\varepsilon_{1, \mathrm{~T}}$ at the $\mathrm{PR}$ is given by

$$
\varepsilon_{1, \mathrm{~T}}=\varepsilon_{1}+\left(1-\varepsilon_{1}\right)(1-\exp (-\lambda C))
$$

From these equations, we may make the following key observations:

- Eqn. (3.10): The maximum density of STs is an increasing function of $\frac{\Delta \varepsilon_{1, \text { th }}}{1-\varepsilon_{1}}$, which is dependent on the type of PN services and the additional outage tolerance.

- Eqn. (3.10): For the same additional outage constraint $\Delta \varepsilon_{1, \text { th }}$ and $C$, spectrum sharing gains are greater for the PN which experiences higher initial outage.

- Eqn. (3.11): The additional outage at the PR due to a $\mathrm{SN}$ is a decreasing function of initial outage.

Consider the following case for certain topologies of interferers (in our case, its STs) discussed in literature where the STs are distributed around the finite circular or infinite area around the typical PR with or without an exclusion region. The purpose 


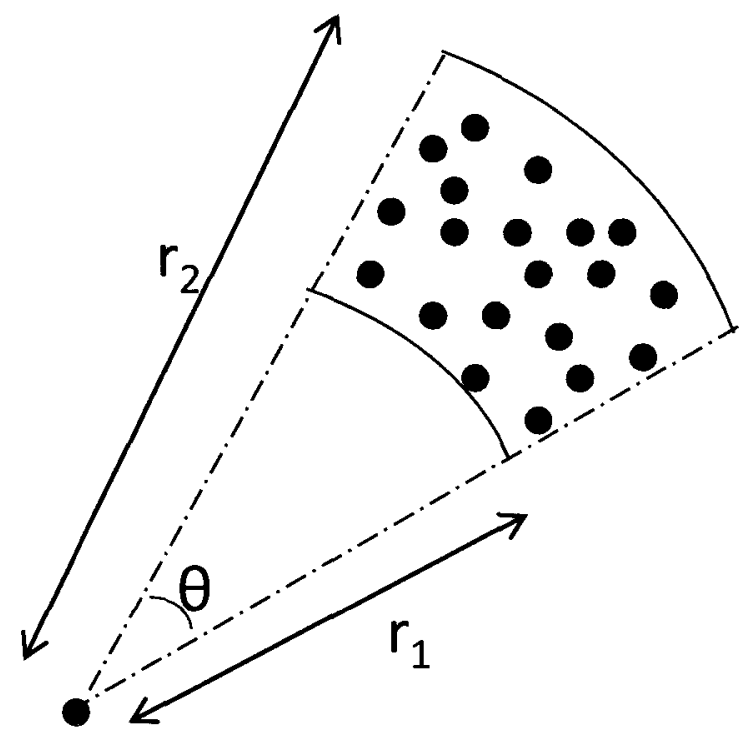

Figure 3.2: STs distributed over a sector around the PR.

of the exclusion region around the PR is to alleviate the profound effect of the nearby interfering transmissions.

\section{SN Distribution: HPPP over an Annular Sector}

Let $\Pi$ be an HPPP with density $\lambda$ with ST transmit power $\rho_{\mathrm{t}}$ over a sector $S$, subtending an angle $\theta$ at the PR and between radii $r_{1}$ and $r_{2}$ as shown in Fig. (3.2). We can write $(3.9)$ as

$$
\begin{aligned}
C & =\theta \int_{r_{1}}^{r_{2}} \frac{r \mathrm{~d} r}{1+r^{\alpha} / \kappa \rho_{\mathrm{t}}} \\
& =\theta\left(p\left(r_{2}\right)-p\left(r_{1}\right)\right)
\end{aligned}
$$

where

$$
p(r)=\int_{0}^{r} \frac{r \mathrm{~d} r}{1+r^{\alpha} / \kappa \rho_{\mathrm{t}}} .
$$


Substituting $x=r^{\alpha}, \beta=1 / \kappa_{1} \rho_{\mathrm{t}}, \nu=n=1, \mu=2 / \alpha$ in (3.194.1) and (3.194.4) of [40] gives

$$
p(r)= \begin{cases}\frac{1}{2} r^{2}{ }_{2} F_{1}\left(1, \frac{2}{\alpha} ; 1+\frac{2}{\alpha} ; \frac{-r^{\alpha}}{\kappa_{1} \rho_{\mathrm{t}}}\right), & 0<r<\infty \\ 0 & r=0 \\ \frac{\pi\left(\kappa_{1} \rho_{t}\right)^{\frac{2}{\alpha}}}{\alpha \sin (2 \pi / \alpha)}, & r \rightarrow \infty,\end{cases}
$$

where ${ }_{2} F_{1}$ is the Gauss-hypergeometric function [40].

Note that the following different topologies are covered by (3.13) and (3.15):

- $\Pi$ is an HPPP over an infinite area around the PR, i.e., $\theta=2 \pi, r_{1}=0, r_{2} \rightarrow \infty$, $[20]$.

- $\Pi$ is an HPPP over infinite area around the victim PR with exclusion region, i.e., $\theta=2 \pi, 0<r_{1}<r_{2}, r_{2} \rightarrow \infty$, [11,32, 41,42].

- $\Pi$ is an HPPP finite area around the PR with or without exclusion region, i.e., $\theta=2 \pi, 0 \leq r_{1}<r_{2}<\infty,[21,43]$.

In order to show the effect of initial outage in the spectrum sharing gains, and to validate the analytical results derived against simulation, we consider the case of STs distributed in a sector region. We plot the maximum density of STs analytically from (3.10) and (3.13), for different additional outage constraints $\Delta \varepsilon_{1, \text { th }}$ as shown in Fig. 3.3. We simulate the interference plus noise $\left(I_{1, \mathrm{p}}+\eta_{1}\right)$ from the PN: its distribution is assumed as the gamma distribution with scale parameter 2 . We tune the shape parameter to obtain different initial outages of study. The gamma distribution is only taken as an example: since our results are only dependent on the initial outage $\varepsilon_{1}$, we may in fact take any distribution to model the sum of the PN interference and noise. We compare the spectrum sharing gains in density of STs for various initial outages. We observe that the actual spectrum sharing gains that can be achieved differ significantly when the initial outage is considered, and hence initial outage cannot be 


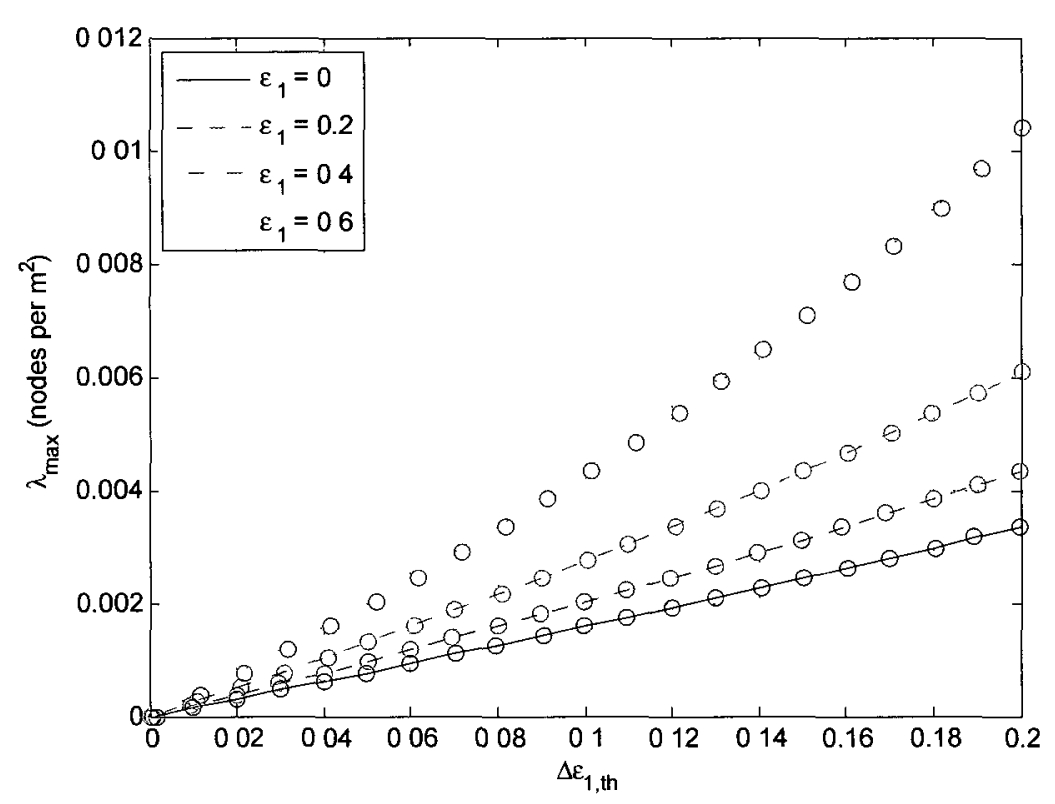

Figure 3.3: Maximum density of STs $\left(\lambda_{\max }\right)$ vs. additional outage constraint $\left(\Delta \varepsilon_{1, \mathrm{th}}\right)$. Lines correspond to analysis, while circles represent simulation with $r_{1}=10 \mathrm{~m}, r_{2}=50$ $\mathrm{m}, \theta=\pi / 3, \alpha=3, \rho_{1, \mathrm{r}}=1 \mu \mathrm{W}, \beta_{1}=10$, and $\rho_{\mathrm{t}}=0.1 \mathrm{~mW}$.

ignored. Furthermore, we notice from the simulations as well as the analytical results that the spectrum sharing gains are greater for those PNs that experience higher initial outage, given the same additional outage constraint. For example, it can be observed in Fig. 3.3 that an additional outage constraint $\Delta \varepsilon_{1, \text { th }}=0.18$ for initial outage $\varepsilon_{1}=0.6$ results in an increase in $\lambda_{\max }$ of approximately $200 \%$ and $66 \%$ as compared to the cases of $\varepsilon_{1}=0$ and $\varepsilon_{1}=0.4$, respectively.

\subsubsection{Effect of Field Size on the Spectrum Sharing Opportunities}

There is a spectrum sharing opportunity for the SN if the additional outage caused by the $\mathrm{SN}$ is below some given outage threshold value $\Delta \varepsilon_{1, \text { th }}$ set by the PR. Using (3.6), (3.8), and (3.13), we can write

$$
p\left(r_{2}\right)-p\left(r_{1}\right) \leq-\frac{1}{\lambda \theta} \log \left(1-\frac{\Delta \varepsilon_{1, \mathrm{th}}}{1-\varepsilon_{1}}\right)
$$




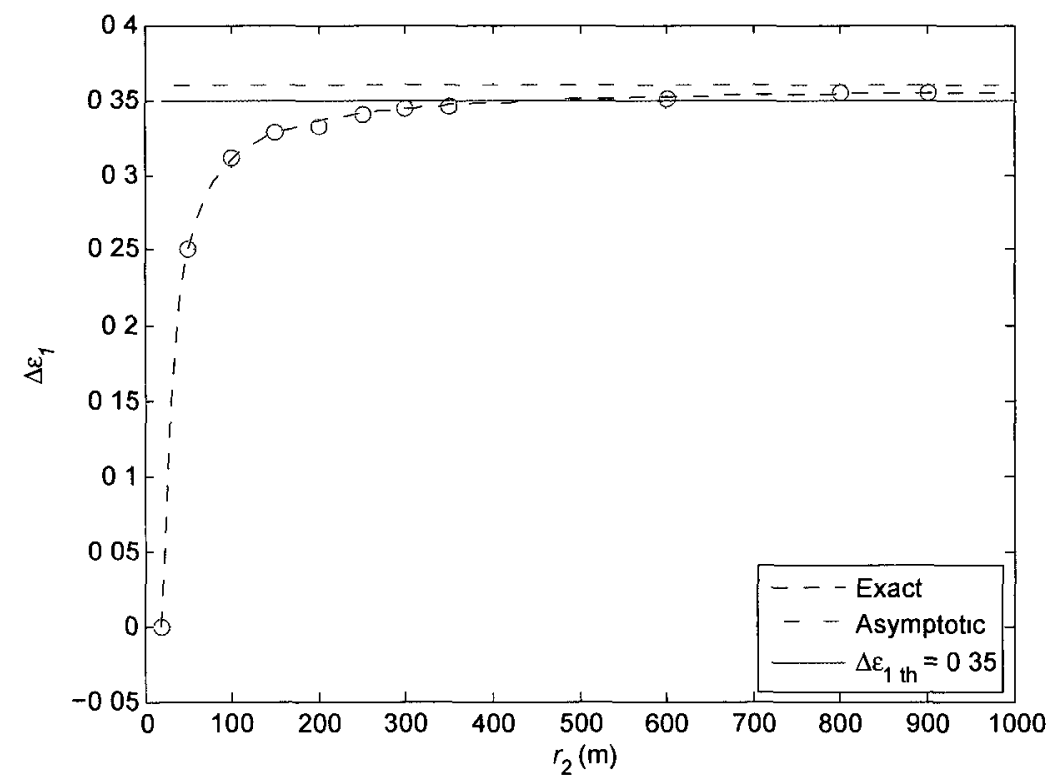

Figure 3.4: Additional outage $\left(\Delta \varepsilon_{1}\right)$ vs. outer field radius $\left(r_{2}\right)$. Lines correspond to analysis, while circles represent simulation, with $r_{1}=20 \mathrm{~m}, \lambda=0.003 / \mathrm{m}^{2}, \theta=2 \pi$, $\varepsilon_{1}=0.4, \Delta \varepsilon_{1, \mathrm{th}}=0.35, \alpha=3, \rho_{1, \mathrm{r}}=1 \mathrm{\mu W}, \beta_{1}=10$, and $\rho_{\mathrm{t}}=0.1 \mathrm{~mW}$.

The set of $\left(r_{1}, r_{2}\right)$ that satisfy the above constraint gives the feasible field size of STs under the given additional outage constraint $\Delta \varepsilon_{1, \text { th }}$. Using (3.14), it can be easily shown that $p(r)$ is a strictly increasing function of $r$. If the $\mathrm{SN}$ is distributed over a finite region between $r_{2}^{*}$ and $r_{1}^{*}$, it is possible that

$$
p\left(r_{2}^{*}\right)-p\left(r_{1}^{*}\right) \leq-\frac{1}{\lambda \theta} \log \left(1-\frac{\Delta \varepsilon_{1, \mathrm{th}}}{1-\varepsilon_{1}}\right)<p(\infty)-p\left(r_{1}^{*}\right)
$$

Hence, assuming an infinite field size when the actual SN is distributed over a finite area can lead to the loss of spectrum sharing opportunities, as indicated by [21]. The effect of assuming an infinite field size in spectrum sharing opportunities is shown in Fig. 3.4 based on (3.16). Spectrum sharing opportunities can be lost by assuming an infinite field size if the actual outer radius of the $\mathrm{SN}$ is less than $450 \mathrm{~m}$ in this case. 


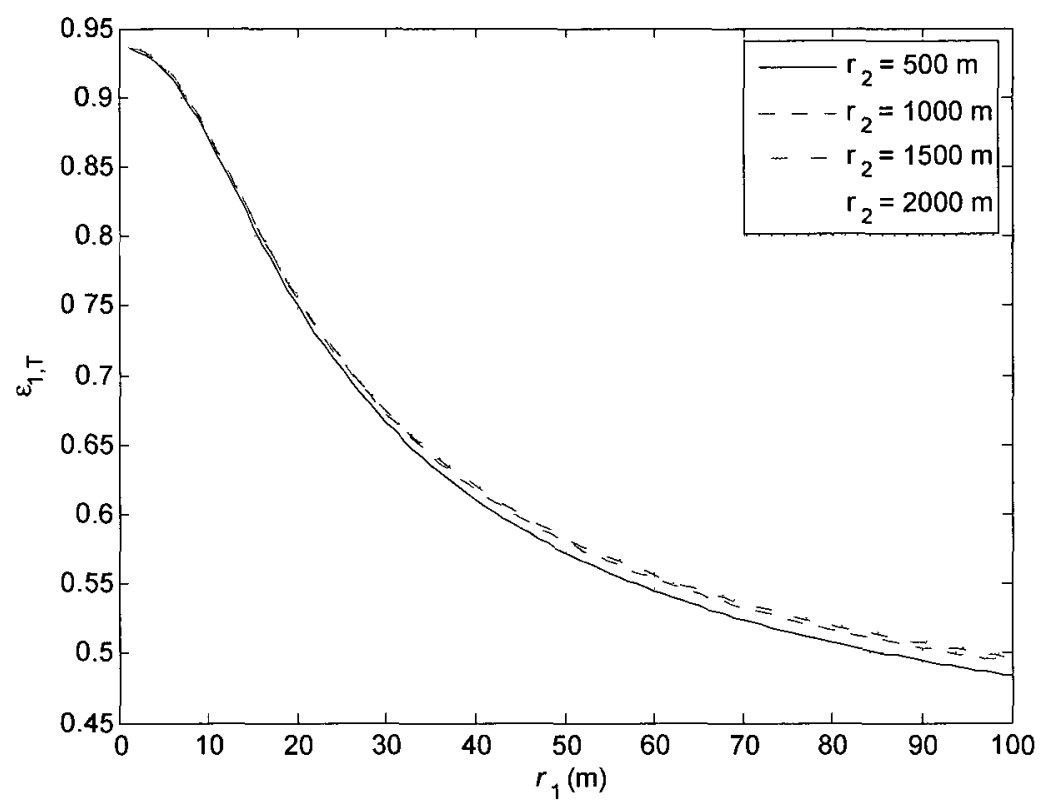

Figure 3.5: Total outage $\varepsilon_{1, \mathrm{~T}}$ vs. inner field radius or exclusion region $r_{1}$ for different outer radius $r_{2}$, with $\lambda=0.003 / \mathrm{m}^{2}, \varepsilon_{1}=0.4, \alpha=3, \rho_{1, \mathrm{r}}=1 \mu \mathrm{W}, \beta_{1}=10, \rho_{\mathrm{t}}=0.1 \mathrm{~mW}$.

\section{Effect of Inner Radius $r_{1}$ (Exclusion Region or Guard Zone) on the Outage}

There have been several past works on the effect of exclusion region on the outage suffered by the victim receiver $[11,15,23,42]$. Exclusion region or guard zone is the minimum distance up to which interferer can come close to the victim receiver. This guard zone is proposed to reduce the profound effect of the nearby interferers. The closed-form equation derived for the finite region gives the effect of the exclusion region accurately. From Fig. 3.5, we observe that the inner radius $r_{1}$ has a profound effect on the outage experienced by the PR. Even a small change in the inner radius has more dominant effect on the outage than the significant change in the outer radius $r_{2}$. 


\section{Convergence in Outage to a value $<1$, wrt to Field Size}

The total outage $\varepsilon_{1, \mathrm{~T}}$ as a function of the outer field radius $r_{2}$ can be written as $\varepsilon\left(r_{2}\right)$. We find the limit $\varepsilon_{1, \mathrm{~T}}\left(r_{2}\right)$ as $r_{2} \rightarrow \infty$ as:

$$
\lim _{r_{2} \rightarrow \infty} \varepsilon_{1, \mathrm{~T}}\left(r_{2}\right)=\left(1-\varepsilon_{1}\right)\left(1-\exp \left(-\lambda \theta\left(\frac{\pi\left(\kappa_{1} \rho_{\mathrm{t}}\right)^{\frac{2}{\alpha}}}{\alpha \sin \left(\frac{2 \pi}{\alpha}\right)}-p\left(r_{1}\right)\right)\right)\right) .
$$

This is in compliance with the previous studies $[9,10]$ that the aggregate interference power converges with the field size, given that the $\alpha>2$. In certain cases, even if the outer radius is infinite, the outage constraint can still be satisfied if the exclusion radius satisfies (using (3.15) and (3.16))

$$
\begin{gathered}
r_{1} \geq p^{-1}\left(\frac{\pi\left(\kappa_{1} \rho_{\mathrm{t}}\right)^{\frac{2}{\alpha}}}{\alpha \sin \left(\frac{2 \pi}{\alpha}\right)}+\frac{1}{\lambda \theta} \log \left(1-\frac{\Delta \varepsilon_{1, \mathrm{th}}}{1-\varepsilon_{1}}\right)\right), \\
\quad \text { for } \quad \frac{\pi\left(\kappa_{1} \rho_{\mathrm{t}}\right)^{\frac{2}{\alpha}}}{\alpha \sin \left(\frac{2 \pi}{\alpha}\right)}+\frac{1}{\lambda \theta} \log \left(1-\frac{\Delta \varepsilon_{1, \mathrm{th}}}{1-\varepsilon_{1}}\right) \geq 0 .
\end{gathered}
$$

\subsubsection{Deployment of Maximum Number of STs}

It can also be interesting to study how many STs can be deployed over a given sector area. The number of nodes is itself a Poisson random variable, whose mean is given by $N=\left(\frac{1}{2} \lambda \theta\left(r_{2}^{2}-r_{1}^{2}\right)\right)$. Now for a given $\left(r_{1}, r_{2}\right)$, using (3.10) and (3.13), the maximum $N$ is given by

$$
N_{\max }=\frac{-\log \left(1-\frac{\Delta \varepsilon_{1, \mathrm{th}}}{1-\varepsilon_{1}}\right)\left(r_{2}^{2}-r_{1}^{2}\right)}{2\left(p\left(r_{2}\right)-p\left(r_{1}\right)\right)} .
$$

Conversely, for a given $N_{\max }$, we can find the set of $\left(r_{1}, r_{2}\right)$ that gives the spatial deployment possibilities under the given outage constraint. We plot the maximum average number of the STs $N_{\max }$ against the field size in Fig. 3.6 using (3.20), with the same simulation parameters as in the previous figure. Also, for a given $N_{\max }$, the 


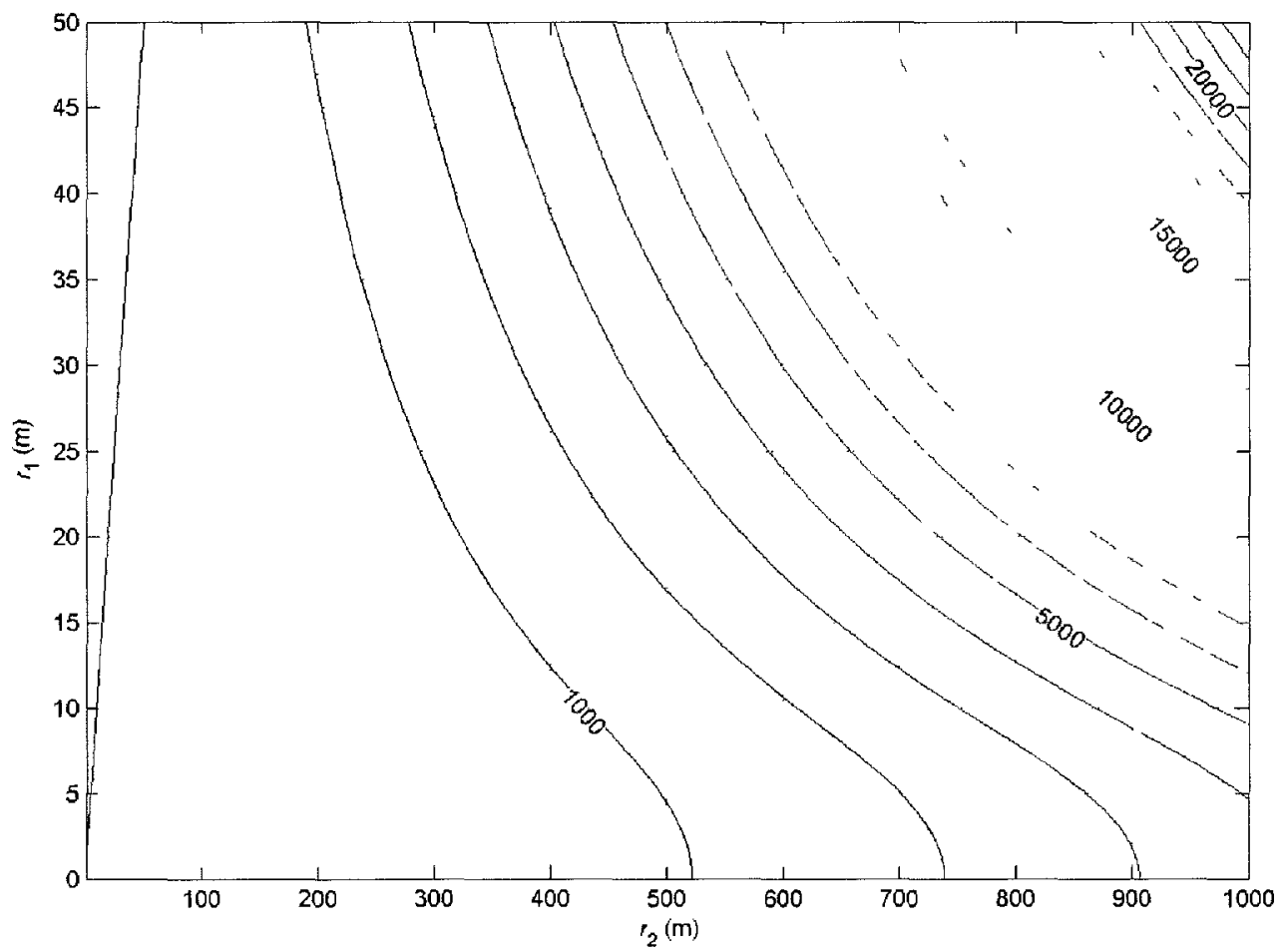

Figure 3.6: Maximum average number of STs $\left(N_{\max }\right)$ vs. field radii $\left(r_{1}, r_{2}\right)$, with $\lambda=0.003 / \mathrm{m}^{2}, \varepsilon_{1}=0.4, \Delta \varepsilon_{1, \mathrm{th}}=0.35, \alpha=3, \rho_{1, \mathrm{r}}=1 \mathrm{\mu W}, \beta_{1}=10, \rho_{\mathrm{t}}=0.1 \mathrm{~mW}$. Shaded region corresponds to infeasible region, i.e., $r_{1} \geq r_{2}$. 


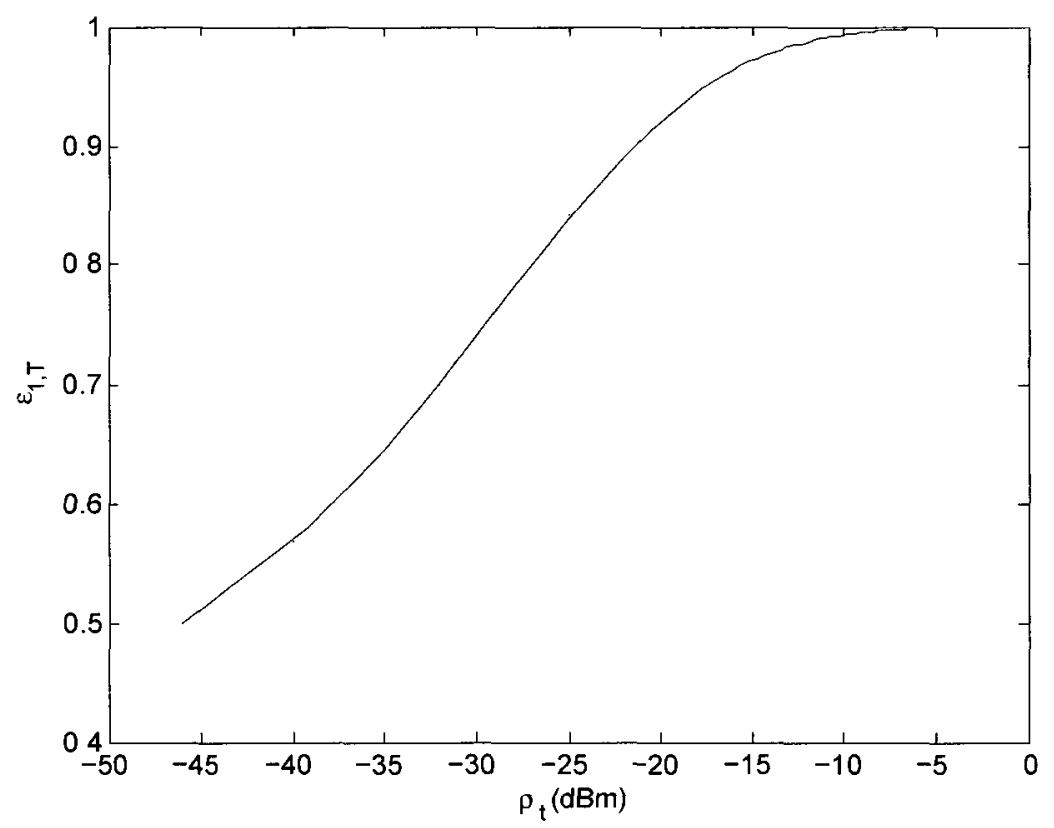

Figure 3.7: Total outage $\left(\varepsilon_{1, \mathrm{~T}}\right)$ vs. ST transmit power $\rho_{\mathrm{t}}$, with simulation parameters: $r_{1}=10 \mathrm{~m}, r_{2}=1000 \mathrm{~m}, \theta=\pi / 3, \alpha=3, \rho_{1, \mathrm{r}}=1 \mu \mathrm{W}, \beta_{1}=10, \lambda=0.003 / \mathrm{m}^{2}$.

contour plot shows the possible solutions for $\left(r_{1}, r_{2}\right)$.

\section{Effect of SU transmit power $\rho_{\mathrm{t}}$ on the outage}

Apart from the density $\lambda$ of SUs, the spectrum sharing gains can also be expressed through the transmit power $\rho_{\mathrm{t}}$ of the STs. The effect of the density of STs on the outage has already been shown in the Fig. 3.3. Here, we plot the outage at the PR with the transmit power $\rho_{\mathrm{t}}$ of the ST as shown in Fig. 3.7.

\subsubsection{Bounded and Unbounded Path-loss models}

In our work, we have taken unbounded attenuation model $g(r)=r^{-\alpha}$ which suffers from singularity at $r=0$. However, in the case of exclusion region $(>1)$, the effect of the singularity gets removed. The effect of singularity on the aggregate interference and the network performance parameters like BER has been discussed in [44]. They studied the case of infinite field-size and drew conclusions. We would like to compare 


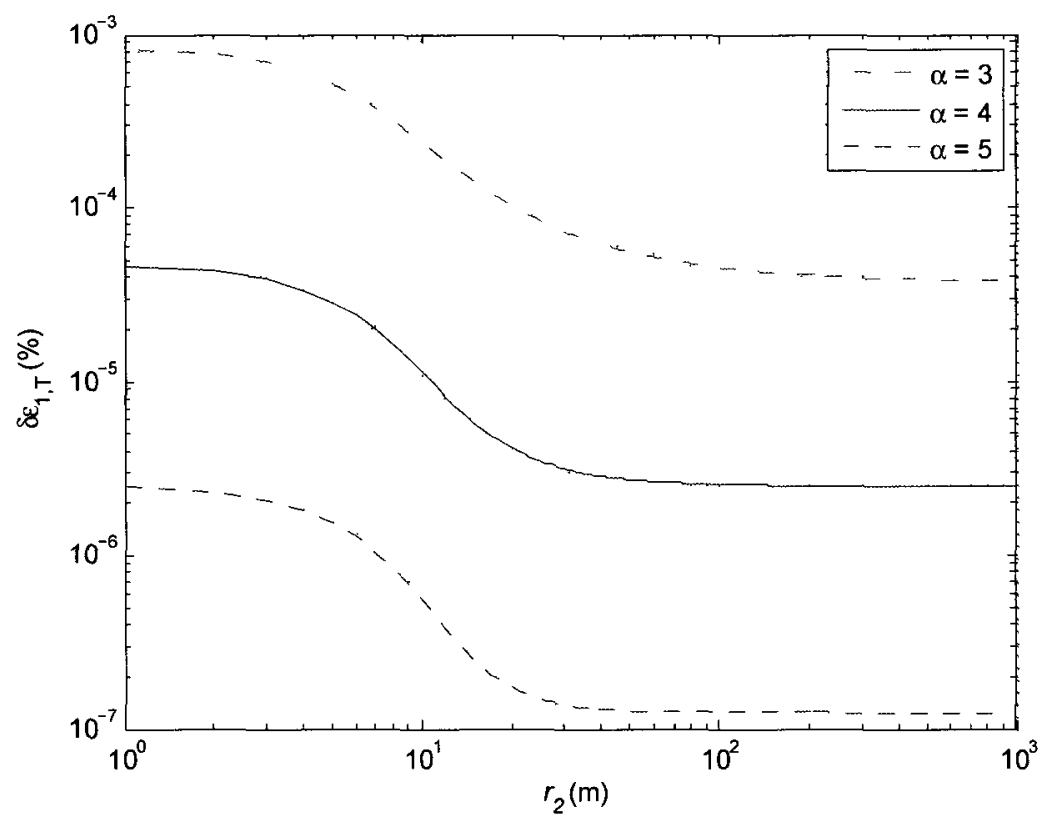

Figure 3.8: Percentage increase in total outage $\left(\delta \varepsilon_{1, \mathrm{~T}}\right)$ vs. field radius $r_{2}$, when unbounded path-loss model is used over bounded path-loss model, for different pathloss exponent $\alpha$ with simulation parameters $r_{0}=1 \mathrm{~m}, \theta=2 \pi, \rho_{1, \mathrm{r}}=1 \mathrm{\mu W}, C_{0}=1, \rho_{\mathrm{t}}=0.1$ $\mathrm{mW}, \beta=10, \lambda=0.003 / \mathrm{m}^{2}$.

the outages from bounded and unbounded path-loss models for different parameters.

\section{Unbounded path-loss model}

In this case, $r_{1}=0$ with $r_{2}>0$. Hence the total outage $\varepsilon_{1, \mathrm{~T}}$ is given by

$$
\varepsilon_{1, \mathrm{~T}}=\varepsilon_{1}+\left(1-\varepsilon_{1}\right)\left(1-\exp \left(-\lambda \theta p\left(r_{2}\right)\right)\right)
$$




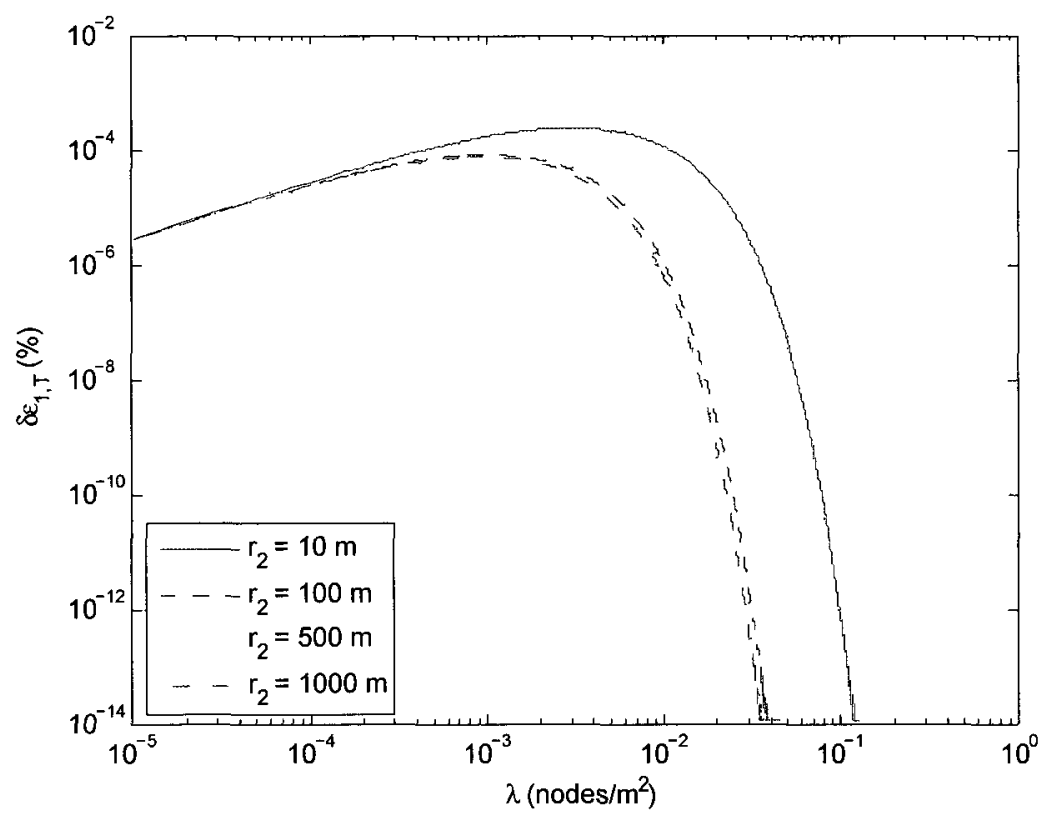

Figure 3.9: Percentage increase in total outage $\left(\delta \varepsilon_{1, \mathrm{~T}}\right)$ vs. density of STs $\lambda$, when unbounded path-loss model is used over bounded path-loss model, for different fieldsize $r_{2}$ with simulation parameters $r_{0}=1 \mathrm{~m}, \alpha=3, \theta=2 \pi, \rho_{1, r}=1 \mu \mathrm{W}, C_{0}=1, \rho_{\mathrm{t}}=0.1$ $\mathrm{mW}, \beta_{1}=10$.

\section{Bounded path-loss model}

We take a well-accepted unbounded path-loss model $g(r)$ as taken in [43] and also verified empirically in [45]:

$$
g(r)= \begin{cases}C_{0}, & r<r_{0} \\ r^{-\alpha} & r \geq r_{0}\end{cases}
$$

where $C_{0}$ is some constant. Now let $I_{1, s}=I_{1}+I_{2}$, where $I_{1}$ and $I_{2}$ denotes the interference coming from inside the two-dimensional ball $b_{2}\left(0, r_{0}\right)$ and outside the ball $b_{2}\left(0, r_{0}\right)$, i.e., $b_{2}\left(0, r_{2}\right) / b_{2}\left(0, r_{0}\right)$. Since both $I_{1}$ and $I_{2}$ are independent, we can write $\mathcal{L}_{I_{1, s}}(s)=\mathcal{L}_{I_{1}}(s) \mathcal{L}_{I_{2}}(s)$. Using Campbell theorem (3.4) and after some manipulations 
we find the total outage $\varepsilon_{1, \mathrm{~T}}^{\prime}$ as

$$
\varepsilon_{1, \mathrm{~T}}^{\prime}=\varepsilon_{1}+\left(1-\varepsilon_{1}\right)\left(1-\exp \left(-\lambda \theta\left(p\left(r_{2}\right)-p\left(r_{0}\right)+\frac{1}{2} \frac{r_{0}^{2} \kappa_{1} C_{0} \rho_{\mathrm{t}}}{\kappa_{1} C_{0} \rho_{\mathrm{t}}+r_{0}^{2}}\right)\right)\right)
$$

Hence, the error in the outage probabilities $\varepsilon_{1, \mathrm{~T}}-\varepsilon_{1, \mathrm{~T}}^{\prime}$ from two path-loss models is given by

$$
\varepsilon_{1, \mathrm{~T}}-\varepsilon_{1, \mathrm{~T}}^{\prime}=\left(1-\varepsilon_{1}\right)\left(\exp \left(-\lambda \theta\left(p\left(r_{2}\right)-p\left(r_{0}\right)+\frac{1}{2} \frac{r_{0}^{2} \kappa_{1} C_{0} \rho_{\mathrm{t}}}{\kappa_{1} C_{0} \rho_{\mathrm{t}}+r_{0}^{2}}\right)\right)-\exp \left(-\lambda \theta p\left(r_{2}\right)\right)\right)
$$

We find the percentage increase in the total outage due to the non-singular pathloss model from the singular path-loss model, i.e., $\delta \varepsilon_{1, \mathrm{~T}}=\frac{\varepsilon_{1, \mathbf{T}}-\varepsilon_{1, \mathrm{~T}}^{\prime}}{\varepsilon_{1, \mathrm{~T}}^{\prime}} \times 100 \%$, and plot it against the field size $r_{2}$. As shown in Fig. 3.8, the percentage increase in the total outage $\delta \varepsilon_{1, \mathrm{~T}}$ takes significantly small values. For $\alpha>2, \delta \varepsilon_{1, \mathrm{~T}}$ decreases with the increase in the field size $r_{2}$, and converges to a finitely small value with $r_{2} \rightarrow \infty$. Hence, even though interference is unbounded when unbounded path-loss model is used over bounded path-loss model, the outage differences are significantly small.

From Fig. 3.9, it is interesting to observe the behaviour of $\delta \varepsilon_{1, \mathrm{~T}}$ with the density of the interferers (here, STs). Similar to before, again $\delta \varepsilon_{1, \mathbf{T}}$ takes small values. At first, $\delta \varepsilon_{1, \mathrm{~T}}$ increases with an increase in the density and then after reaching maximum value, it starts decreasing with the density. Or, there exist a density of STs at which $\delta \varepsilon_{1, \mathrm{~T}}$ is maximum. Hence, we cannot say that inaccuracy in the total outage due to unbounded path-loss model increases or decreases with the density of interferers.

\subsection{Summary}

The result derived in (2.11) is applied in the spectrum sharing scenario to find the additional outage at a PR of a PN due to the deployment of a SN. The SN must be designed to satisfy (3.6) in order to share the spectrum of PU. Through the closed-form 
equations derived, the actual problem of interest in the spectrum sharing scenarios is addressed. We have analyzed the spectrum sharing gains for an additional outage constraint given by the typical PR, that are shown to be significantly dependent on the initial outage condition (Fig. 3.3). In addition, the results are extended to derive a closed-form expression for a finite annular sector field of interferers (or, STs), that include the cases with or without an exclusion region around the typical PR. Using this result, we analyzed the effect of the field size on the spectrum sharing opportunities and gains (Figs. 3.4, 3.6). Conditions are shown where an assumption of an infinite field size can or cannot lead to loss in spectrum sharing opportunity. In some studies on infinite field size, simulating infinite region is itself a problem. Results derived for finite annular region can be used to validate that the finite region taken for simulation is a close approximation to the infinite field size. It is worth mentioning that these results also include PN interference. Finally, we also analyzed the effect of ST power $\rho_{\mathrm{t}}$ on the outage. Outages are compared in case of unbounded and bounded path-loss models for different field sizes and densities, and the difference is found to be significantly small. Further, there exists a density at which the difference in the outage values is maximum.

This analysis has the advantage of being simple and exact, and can be used in practical scenarios'with complex PNs (where nodes are coordinating in complex ways) to get insights into the spectrum sharing opportunities and gains. The closed-form equations are derived for obtaining the outage at a receiver due to the interferers distributed over a finite field. The $\mathrm{SN}$ is a general term which can be a single user or a group of users. This analysis is equally applicable for the case of unlicensed spectrum sharing networks, other combinations of heterogeneous networks or even the sets of interferers in same network. 


\section{Chapter 4}

\section{Cellular Network Overlaid by an Ad hoc Network Incorporating the Sensing Mechanism: The Uplink}

\section{Case}

\subsection{Introduction}

Owing to the spectrum scarcity and inefficient spectrum use, some spectrum sharing scenarios have been discussed in the literature where a SN shares the spectrum of a PN. The most common scenario is the ad hoc network overlaid over the cellular network. Due to the possible additional outage tolerance available in the cellular network, the ad hoc network can share the spectrum under the given outage constraint by the cellular network in an underlay scheme [31].

In many cases $[26,37,46]$, where multiple PRs are considered, $\mathrm{PN}$ is assumed to be distributed as a PPP which can be quite a narrow assumption, especially for the cellular network. There are always complex ways through which the primary nodes in the cellular network are distributed. Moreover, there is always an inherent separation between the co-channel devices, especially, in the cellular network and hence the cellular network cannot be accurately modeled as PPP. For instance, in [26], multiple PRs and PTs are taken into account but again the cellular BSs and users are assumed 
to be distributed as a PPP. Additionally, in these works, PRs are assumed to have identical system parameters and outage requirements, which does not accommodate diverse primary user services.

As discussed in the previous chapter, a presumably exact exclusion region (or guard zone) around a typical PR has been considered to reduce the profound effect of the nearby STs $[11,15]$. While this kind of exclusion region is possible by drawing a physical circular boundary, however, guaranteeing perfect exclusion region through sensing channels is impractical in the fading environment. Previously, sensing mechanism has been mostly used in the overlay scheme to detect the presence of PR [31], except in [12], where the outage is studied at the single PR by incorporating sensing with the randomly distributed STs, using lognormal fit for the interference. In [20], sensing mechanism has been considered to detect the presence of the PR under the given outage constraint by a single PR. However, in this work, sensing of the PR at every ST is taken independently of its distance from the PR.

We extend the outage analysis for the single PR done in the previous chapter to the case of randomly but not necessarily independently distributed cellular PRs. In this chapter, we make use of the sensing mechanism rather than the presumably exact exclusion region to decrease the profound effect of the nearby STs and accordingly, an upper bound for the outage experienced by the PRs is derived. This bound is tight where the co-channel PRs are spatially separated by relatively significant distances, which is the case in the cellular network. Sensing mechanism is shown to be quite significant in decreasing the outage suffered by PRs. For the maximum spectrum sharing gains, we optimize the decision threshold (to decide whether to transmit or not) used by the STs under the given outage constraints. Additionally, in our study, every co-channel primary signal link may have different system parameters. We confirm the analytical results derived through Monte Carlo simulations. Furthermore, we show that the fading in the sensing channels serves as a boon in increasing the 


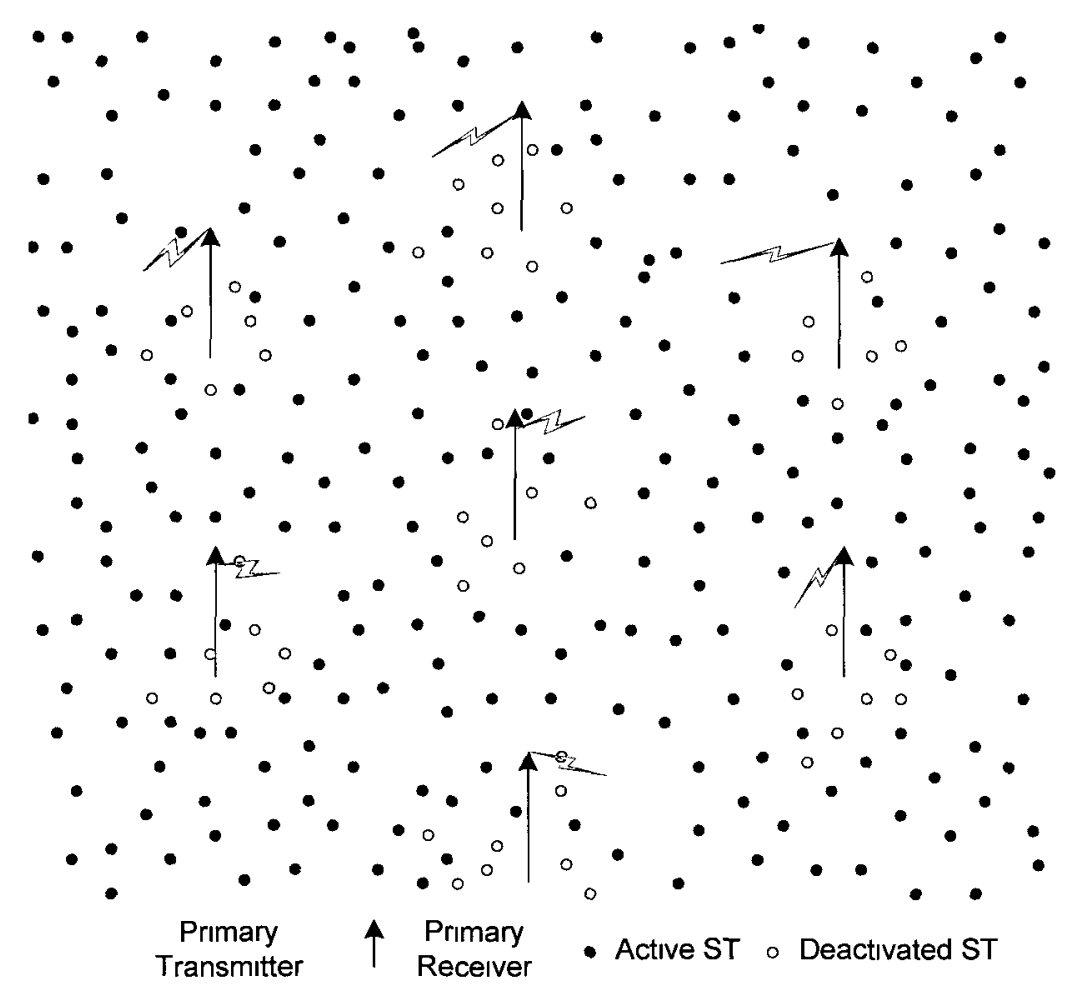

Figure 4.1: Illustration of the cellular network sharing spectrum with the secondary ad hoc network in the uplink case.

spectrum sharing gains. It is shown that in this case the spectrum sharing gains are more than the case when a presumably exact circular exclusion region is used.

\subsection{System Model}

Consider a setup where a secondary ad hoc network is overlaid on the primary cellular network as shown in Fig. 4.1. Let the locations of these co-channel PRs (or basestations (BSs)) be distributed randomly over $S \subseteq \mathbb{R}^{2}$ denoted by a point process $\Pi_{p}=$ $\left\{Y_{1}, Y_{2}, \ldots\right\} . \Pi_{\mathrm{p}}$ can follow any real unknown distribution characterizing the locations of the PRs where the PRs are inherently separated by relatively large distances. STs are assumed to be distributed as the points of a PPP $\Pi_{s}=\left\{X_{1}, X_{2}, \ldots.\right\}$ over $\mathbb{R}^{2}$ with density $\lambda$ and transmit power $\rho_{\mathrm{t}}$. We assume the location $Y_{\imath}$ of the $\imath^{\text {th }} \mathrm{PR}$ of study to 
be at the origin in the polar coordinate system. Accordingly, we define a new point process $\Pi_{\imath, \mathrm{p}}=\left\{Y_{1, \imath}, Y_{2, \imath}, \ldots\right\} \subset \mathbb{R}^{2}$, conditioned on the location of the $\imath^{\text {th }} \mathrm{PR}$, where $Y_{k, \imath}=Y_{k}-Y_{\imath}$ denotes the location of the $k^{\text {th }}$ PR w.r.t $\imath^{\text {th }}$ PR.

We study the outage at the $i^{\text {th }}$ PR due to the PN interference as well as the SN interference. For a given useful signal link distance $d_{\imath}$, the signal received at this $i^{\text {th }}$ $\mathrm{PR}$ is given by

$$
S_{\imath}=\gamma_{\imath} \rho_{\imath, \mathrm{r}}\left(d_{\imath}\right)
$$

where the $d_{\imath}$ is the PR-PT distance at desired signal link. $\rho_{\imath, \mathrm{r}}\left(d_{\imath}\right)$ is the mean received power by the transmitter of this PR at the desired signal link, $\gamma_{2}$ is the exponentially distributed random variable representing Rayleigh fading on the desired signal link. For full large-scale path-loss compensation, i.e., constant large-scale received power, we have $\rho_{\imath, \mathrm{r}}\left(d_{\imath}\right)=\rho_{\imath, \mathrm{r}} \forall i$, as utilized by the modern cellular standards, for the uplink case. In the absence of the SN, let the unwanted signal power at the $\imath^{\text {th }} \mathrm{PR}$ be $I_{\imath, \mathrm{p}}+\eta_{\imath}$, accounting for both the interference $I_{\imath, \mathrm{p}}$ coming from the co-channel PN interferers (PTs) as well as the noise $\eta_{\imath}$. The corresponding initial outage probability $\varepsilon_{\imath}\left(d_{\imath}\right)$ at this $\mathrm{PR}$ is given by

$$
\mathcal{P}\left(\frac{S_{\imath}}{I_{\imath, \mathbf{p}}+\eta_{\imath}}<\beta_{\imath}\right)=\varepsilon_{\imath}\left(d_{\imath}\right)
$$

where $\beta_{\imath}$ is the SINR threshold. Now, in the presence of SN, the net unwanted signal at the $i^{\text {th }}$ victim PR is $I_{\imath, \mathrm{p}}+I_{\imath, \mathrm{s}}+\eta_{\imath}$, where $I_{\imath, \mathrm{s}}$ is the interference coming from the $\mathrm{SN}$. The corresponding total outage $\varepsilon_{\imath, \mathrm{T}}\left(d_{\imath}\right)$ experienced by this $i^{\text {th }} \mathrm{PR}$ is given by

$$
\mathcal{P}\left(\frac{S_{\imath}}{I_{\imath, \mathrm{p}}+I_{\imath, \mathrm{s}}+\eta_{\imath}}<\beta_{\imath}\right)=\varepsilon_{\imath, \mathrm{T}}\left(d_{\imath}\right)
$$

As done before, we use the marked Poisson process theory to characterize the STs. We define $\Pi_{\imath, \mathrm{s}}=\left\{\left(X_{\jmath, \imath}, \Gamma_{\jmath, \imath}\right) \mid X_{\jmath} \in \Pi_{s}\right\}$ over the product space $\mathbb{R}^{2} \times \mathbb{R}^{+}$, where $\Gamma_{\jmath, \imath}$ is the mark associated with the interferer at $X_{\jmath, \imath}=X_{\jmath}-Y_{\imath}$, representing fading on 
the interference channel between this $j^{\text {th }}$ interferer and the $i^{\text {th }}$ PR. The marks are distributed identically and independently for every $\jmath$, and independently of $X_{\jmath, \imath}$. Due to the spatial stationarity of $\Pi_{s}$ and from the Marking Theorem [8], $\Pi_{\imath, \mathrm{s}}$ is also a PPP.

\subsection{Outage Analysis}

We begin by studying the outage at the $\imath^{\text {th }}$ PR due to the SN interference $I_{\imath, \mathrm{s}}$ as well as the PN interference $I_{\imath, \mathrm{p}}$. Consider two separate cases : one with and other without the sensing mechanism around the PRs.

\subsubsection{Without Sensing Mechanism}

In this case, there is no sensing mechanism adopted by the STs to sense the PRs. Due to the spatial stationarity property of the HPPP [8], the statistics of the interference coming from the STs at any point are the same, therefore, $I_{\imath, \mathrm{s}}=I_{s} \forall Y_{\imath} \in \Pi_{p}$. Moreover, $I_{\imath, \mathbf{s}}$ and $I_{\imath, \mathbf{p}}$ are independent, and in this case, we can apply the outage equation derived for a single PR (using (2.12)), for the two independent unwanted signal sets, to find the total outage $\varepsilon_{\imath, \mathrm{T}}\left(d_{\imath}\right)$ as

$$
\varepsilon_{\imath, \mathrm{T}}\left(d_{\imath}\right)=1-\left(1-\varepsilon_{\imath}\left(d_{\imath}\right)\right) \mathcal{L}_{I_{\imath, \mathrm{s}}}\left(\kappa_{\imath}\right)
$$

Using (3.8), (3.13), and (3.15) for $r_{1}=0$ and $r_{2} \rightarrow \infty$, the above equation can be expressed as

$$
\varepsilon_{\imath, \mathrm{T}}\left(d_{\imath}\right)=1-\left(1-\varepsilon_{\imath}\left(d_{\imath}\right)\right) \exp \left(\frac{-\lambda 2 \pi^{2}\left(\kappa_{\imath} \rho_{\mathrm{t}}\right)^{\frac{2}{\alpha}}}{\alpha \sin (2 \pi / \alpha)}\right)
$$

where $\kappa_{\imath}=\frac{\beta_{2}}{\rho_{\imath, \mathrm{r}}\left(d_{2}\right)}$. 
Let $\varepsilon_{\imath, \mathrm{T}}=\mathbb{E}_{d_{\imath}}\left(\varepsilon_{\mathrm{T}, \imath}\left(d_{\imath}\right)\right)$ and $\varepsilon_{\imath}=\mathbb{E}_{d_{\imath}}\left(\varepsilon_{\imath}\left(d_{\imath}\right)\right)$, then from (4.5) we have

$$
\varepsilon_{\imath, \mathrm{T}}=1-\left(1-\varepsilon_{\imath}\right) \exp \left(\frac{-\lambda 2 \pi^{2}\left(\kappa_{\imath} \rho_{\mathrm{t}}\right)^{\frac{2}{\alpha}}}{\alpha \sin (2 \pi / \alpha)}\right)
$$

under large-scale power control.

\subsubsection{With Sensing Mechanism}

In order to avoid the nearby secondary transmissions around each PR, we use the sensing mechanism instead of presumably exact exclusion region. We consider distributed sensing for all the PRs, s.t., each ST senses independently of the other STs and decides whether to transmit or not. Sensing is done at the ST through a beacon sent by the PR on an out of band channel, assuming full-duplex channel for the PRs and the STs. Alternatively, sensors at ST can perform the sensing operations. More information about the distributed sensing can be obtained in [4] and the references therein. We assume that the detection of the PR by any ST is independent of the beacons transmitted by the other PRs, since these are low power beacons transmitted by distant PRs. Or, in other words, the sensing channel do not suffer from interference.

We define a set of deactivated STs that are not allowed to transmit around the $k^{\text {th }} \mathrm{PR}$ (at location $Y_{k, \imath}$ ) as

$$
\mathbb{S}_{k, \imath}=\left\{\left(X_{\jmath, \imath}, \Gamma_{\jmath, \imath}\right): \frac{\Gamma_{\jmath, k, \imath}^{\prime} \rho_{\mathrm{b}}\left|X_{\jmath, \imath}-Y_{k, \imath}\right|^{-\alpha}}{\eta}>\beta_{\imath, \mathrm{th}},\left(X_{\jmath, \imath}, \Gamma_{\jmath, \imath}\right) \in \Pi_{\imath, \mathrm{s}}, Y_{k, \imath} \in \Pi_{\imath, \mathrm{p}}\right\}
$$

where $\Gamma_{\jmath, k, \imath}^{\prime}$ is the exponentially random variable, representing Rayleigh fading on the sensing channel between the $k^{\text {th }} \mathrm{PR}$ and the $j^{\text {th }} \mathrm{ST}$, and is independent of $\Gamma_{\jmath, \imath} \cdot \rho_{\mathrm{b}}$ is the probe or beacon power, $\beta_{\imath, \text { th }}$ is the SNR threshold (or, decision threshold) for ST for deciding whether to transmit or not, and $\eta$ is the background noise. Hence, 
the STs that are allowed transmissions can be expressed as

$$
\Pi_{\imath, \mathbf{s}, \mathrm{t}}=\Pi_{\imath, \mathrm{s}} / \cup_{Y_{k, \imath} \in \Pi_{\imath, \mathrm{p}}} \mathbb{S}_{k, \imath}
$$

STs that are able to receive the beacon (transmitted by the PRs) above a certain threshold level are not allowed to transmit, as there are higher chances that they are close to the PRs. The probability of a ST being allowed to transmit is dependent on the positions of the PRs. Hence, the PN interference $I_{\imath, \mathrm{p}}$ and the SN interference $I_{2, \mathrm{~s}}$ are not independent. Due to this interaction between the PRs and STs, or, subsequently between the PTs and STs, it seems infeasible to obtain the exact outage expressions as indicated by [6]. However, we make use of the facts: 1) the SNs are generally dense networks having short transmission ranges 2) the cellular cochannel PRs (especially, in the uplink case) are inherently separated by relatively large distances in the cellular network, to derive the upper bound for the outage experienced by the PRs. This bound is supposed to be tight for the cellular PN and helps us to give the analytical insights of various system parameters.

We can say that for any realization, $I_{\imath, \mathrm{s}}^{+} \geq I_{\imath, \mathrm{s}}$, where $I_{\imath, \mathrm{s}}^{+}$is the interference coming from STs distributed as $\Pi_{\imath, \mathrm{s}, \mathrm{t}}^{\prime}=\Pi_{\imath, \mathrm{s}} / \mathbb{S}_{\imath, \imath}$ and $I_{\imath, \mathrm{s}}$ is the interference coming from $\Pi_{\imath, \mathrm{s}, \mathrm{t}}=\Pi_{\imath, \mathrm{s}} / \cup_{Y_{k, \imath} \in \Pi_{\imath, \mathrm{p}}} \mathbb{S}_{k, \imath}$. Now, if $\varepsilon_{\imath, \mathrm{T}}^{+}\left(d_{\imath}\right)$ is the total outage due to $I_{\imath, \mathrm{s}}^{+}+I_{\imath, \mathrm{p}}$, we can say that $\varepsilon_{\imath, T}\left(d_{\imath}\right) \leq \varepsilon_{\imath, T}^{+}\left(d_{\imath}\right)$.

It is easy to observe that $I_{\imath, \mathrm{p}}$ and $I_{\imath, \mathrm{s}}^{+}$are independent and in this case, as done before, we can write $\varepsilon_{\imath}^{+}\left(d_{\imath}\right)$ as

$$
\varepsilon_{\imath, \mathrm{T}}^{+}\left(d_{\imath}\right)=1-\left(1-\varepsilon_{\imath}\left(d_{\imath}\right)\right) \mathcal{L}_{I_{\imath, \mathrm{s}}^{+}}\left(\kappa_{\imath}\right)
$$


The point process $\Pi_{\imath, \mathrm{s}, \mathrm{t}}^{\prime}$ is obtained by location-dependent thinning (here, deactivation) of $\Pi_{\imath, \mathrm{s}}$ resulting in a non-homogeneous point process of density:

$$
\lambda_{\imath, \mathrm{s}, \mathrm{t}}^{\prime}(r)=\lambda \mathcal{P}\left(\frac{\Gamma_{j, \imath, \imath}^{\prime} \rho_{\mathrm{b}} r^{-\alpha}}{\eta} \leq \beta_{\imath, \mathrm{th}}\right)
$$

where $\mathcal{P}\left(\frac{\Gamma_{j, 2,2}^{\prime} \rho_{\mathrm{b}} r^{-\alpha}}{\eta} \leq \beta_{\imath, \text { th }}\right)$ is the probability that a node at location $(r, \theta)$ gets thinned (or, deactivated).

Now, we use the Campbell's theorem for the marked Poisson process [8] to incorporate the sensing mechanism, and hence, $\mathcal{L}_{I_{s, 2}^{+}}\left(\kappa_{z}\right)$ is obtained as

$$
\mathcal{L}_{I_{\imath, \mathrm{s}}^{+}}\left(\kappa_{\imath}\right)=\exp \left\{\lambda \int_{0}^{2 \pi} \int_{0}^{\infty} \int_{0}^{\infty} \int_{0}^{\infty}\left(e^{-\kappa_{\imath} \Gamma \rho_{\mathrm{t}} r^{-\alpha}}-1\right) \mathcal{P}\left(\frac{\Gamma^{\prime} \rho_{\mathrm{b}} r^{-\alpha}}{\eta} \leq \beta_{\imath, \mathrm{th}}\right) e^{-\Gamma} d \Gamma r d r d \theta\right\},
$$

where $\Gamma$ and $\Gamma^{\prime}$ represents fading on the interference and sensing channels, respectively. Both $\Gamma$ and $\Gamma^{\prime}$ have same distributions as $\Gamma_{\jmath, 2}$ and $\Gamma_{\jmath, l, l}^{\prime}$, respectively. Substituting $x=r^{\alpha}$ in (4.10) and using (3.193.4) and (3.383.10) of [40], we derive closed-form result as $\mathcal{L}_{I_{\imath, \mathrm{s}}^{+}}\left(\kappa_{\imath}\right)=\exp \left(-\lambda \Omega_{\imath}\left(\beta_{\imath, \mathrm{th}}\right)\right)$, where

$$
\Omega_{\imath}\left(\beta_{\imath, \mathrm{th}}\right)=\frac{1}{\alpha} 2 \pi\left(\kappa_{\imath} \rho_{\mathrm{t}}\right)^{\frac{2}{\alpha}}\left(\frac{\pi}{\sin (2 \pi / \alpha)}-e^{\frac{\kappa_{\imath} \rho_{\mathrm{t}} \beta_{2, \mathrm{th}} \eta_{2}}{\rho_{\mathrm{b}}}} \Gamma\left(\frac{2}{\alpha}\right) \Gamma\left(1-\frac{2}{\alpha}, \frac{\kappa_{\imath} \rho_{\mathrm{t}} \beta_{2, \mathrm{th}} \eta_{\imath}}{\rho_{\mathrm{b}}}\right)\right) .
$$

Hence, the upper bound $\varepsilon_{\imath, \mathrm{T}}^{+}\left(d_{\imath}\right)$ on the total outage suffered by the $i^{\text {th }} \mathrm{PR}$ is given by

$$
\varepsilon_{\imath, \mathrm{T}}^{+}\left(d_{\imath}\right)=1-\left(1-\varepsilon_{\imath}\left(d_{\imath}\right)\right) \exp \left(-\lambda \Omega_{\imath}\left(\beta_{\imath, \mathrm{th}}\right)\right)
$$

Now, if $\varepsilon_{\imath}^{+}=\mathbb{E}_{d_{\imath}}\left(\varepsilon_{\imath}^{+}\left(d_{\imath}\right)\right)$ and $\varepsilon_{\imath}=\mathbb{E}_{d_{\imath}}\left(\varepsilon_{\imath}\left(d_{\imath}\right)\right)$, we can write the upper bound $\varepsilon_{\imath, \mathrm{T}}^{+}$ as

$$
\varepsilon_{\imath, \mathrm{T}}^{+}=1-\left(1-\varepsilon_{\imath}\right) \exp \left(-\lambda \Omega_{\imath}\left(\beta_{\imath, \mathrm{th}}\right)\right)
$$

under large-scale power control. 


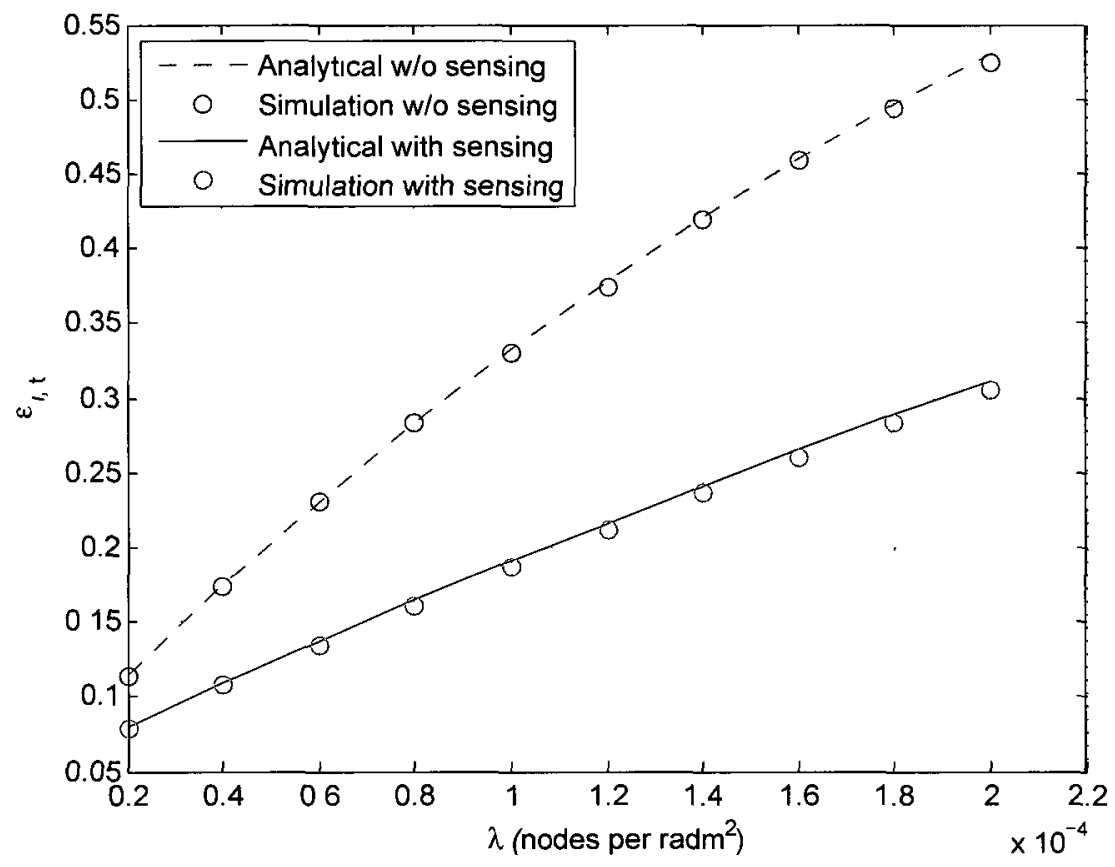

Figure 4.2: Total outage $\varepsilon_{\imath, \mathrm{t}}$ suffered by the $i^{\text {th }} \mathrm{PR}$ with the density of STs $\lambda$ for $\varepsilon_{i}=0.05, \alpha=3, \rho_{\imath, \mathrm{r}}=1 \mathrm{lW}, \beta_{r}=10, \rho_{\mathrm{t}}=1 \mathrm{~mW}, \rho_{\mathrm{b}}=1 \mathrm{~W}$, and $\beta_{\imath, \mathrm{th}}=10$. Distance between the adjacent co-channel BSs is taken as $500 \mathrm{~m}$.

In order to show the effect of sensing on the outage experienced by the PR, and to validate the analytical results (using (4.6) and (4.13)) derived against simulation, we plot (see Fig. 4.2) the total outage $\varepsilon_{\imath, \mathrm{T}}$ suffered by the $i^{\text {th }} \mathrm{PR}$ with the density $\lambda$ of STs. To perform the worst case analysis in case of the sensing mechanism, we take the tier of the co-channel PRs (or BSs) around the $i^{\text {th }}$ PR at a distance of only 500 $\mathrm{m}$ from the $i^{\text {th }} \mathrm{PR}$ and the probe power $\rho_{\mathrm{b}}=1 \mathrm{~W}$. As done in the previous chapter, we simulate the PN interference plus noise $\left(I_{\imath, \mathrm{p}}+\eta_{\imath}\right)$ : its distribution is assumed as the gamma distribution with scale parameter 2. We may even take any other distribution to model the sum of the PN interference and noise, since the results are only dependent on the initial outage $\varepsilon_{\imath}$. We tune the shape parameter of the gamma distribution to obtain any given initial outage $\varepsilon_{\imath}$ of study.

We observe that the upper bound for the outage derived in case of sensing is quite tight in comparison to the Monte Carlo simulation curve. This bound is supposed 
to be tighter in the actual cellular systems where the PRs are inherently separated by the relatively larger distances. Further, we observe from Fig. 4.2 that there is a significant decrease in the total outage suffered by the PR with the increase in the density of STs, when the sensing is used to alleviate the profound effect of nearby interfering transmissions. Conversely, under the given outage constraint, there is a significant increase in the spectrum sharing gains.

\subsubsection{Optimum Decision Threshold}

Now, we want to find the optimum decision threshold $\beta_{\imath \text {,th }}$ for which the maximum spectrum sharing gains (here, the number of STs) are achieved while satisfying the given outage constraint $\varepsilon_{\imath, \text { th }}$ at every primary receiver at $Y_{\imath} \in \Pi_{p}$.

In order to ensure that the outage condition is satisfied, we take $\varepsilon_{\imath, \mathrm{T}}^{+} \leq \varepsilon_{\imath, \mathrm{th}}$. Equivalently, from (4.13), we can write it as

$$
\Omega_{\imath}\left(\beta_{\imath, \mathrm{th}}\right) \leq-\frac{1}{\lambda} \log \left(\frac{1-\varepsilon_{\imath, \mathrm{th}}}{1-\varepsilon_{\imath}}\right) .
$$

Differentiating (4.11), or, from (4.10), it can be easily shown that $\Omega_{\imath}\left(\beta_{\imath, \mathrm{th}}\right)$ is an increasing function of $\beta_{\imath, \mathrm{th}}$. Hence, we can write above equation as

$$
\beta_{\imath, \text { th }} \leq \Omega_{\imath}^{-1}\left(-\frac{1}{\lambda} \log \left(\frac{1-\varepsilon_{\imath, \text { th }}}{1-\varepsilon_{\imath}}\right)\right)
$$

Using (4.7), the number of active STs $N\left(\Pi_{\imath, \mathrm{s}, \mathrm{t}}\right)$ can be expressed as

$$
N\left(\Pi_{\imath, \mathrm{s}, \mathrm{t}}\right)=N\left(\Pi_{\imath, \mathrm{s}}\right)-N\left(\cup_{Y_{k, \imath} \in \Pi_{\imath, \mathrm{P}}} \mathbb{S}_{k, \imath}\right)
$$

In case of cellular primary network, PRs are spatially separated by relatively large distances, and therefore, deactivation of the ST is most likely due to the sensing of 
the nearest PR only (as depicted in the Fig. 4.1). Hence, we can write (4.16) as

$$
\begin{aligned}
& \gtrsim N\left(\Pi_{\imath, \mathrm{s}}\right)-\sum_{Y_{k, \imath} \in \Pi_{\imath, \mathrm{p}}} N\left(\mathbb{S}_{k, \imath}\right)
\end{aligned}
$$

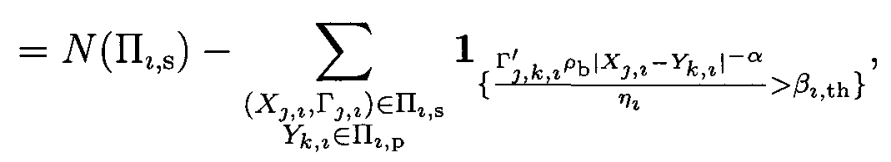

where $1_{\left\{\frac{\Gamma^{\prime} \rho_{\mathrm{b}} r^{-\alpha}}{\eta} \leq \beta_{\imath, \mathrm{th}}\right\}}$ denotes the sensing based indicator variable for the ST at $(r, \theta)$, that decides whether to transmit or not, defined as

$$
\mathbf{1}_{\left\{\frac{\Gamma^{\prime} \rho_{\mathrm{b}} r^{-\alpha}}{\eta} \leq \beta_{\imath, \mathrm{th}}\right\}}= \begin{cases}1, & \frac{\Gamma^{\prime} \rho_{\mathrm{b}} r^{-\alpha}}{\eta} \leq \beta_{\imath, \mathrm{th}} \\ 0, & \frac{\Gamma^{\prime} \rho_{\mathrm{b}} r^{-\alpha}}{\eta}>\beta_{\imath, \mathrm{th}}\end{cases}
$$

From (4.17), the maximum number of active STs can be expressed as

$$
\max _{\beta_{i, \mathrm{th}}}\left\{N\left(\Pi_{\imath, \mathrm{s}, \mathrm{t}}\right)\right\} \equiv \max \left(\beta_{\imath, \mathrm{th}}\right)
$$

which holds true $\forall Y_{\imath} \in \Pi_{\mathrm{p}}$. Hence, using (4.15), the optimum decision threshold $\beta_{\imath \text {,th }}^{\text {opt }}$ is given by

$$
\beta_{\imath, \mathrm{th}}^{\mathrm{opt}}=\Omega_{\imath}^{-1}\left(-\frac{1}{\lambda} \log \left(\frac{1-\varepsilon_{\imath, \mathrm{th}}}{1-\varepsilon_{\imath}}\right)\right), \forall Y_{\imath} \in \Pi_{\mathrm{p}} .
$$

We show the effect of the decision threshold $\beta_{\imath, \text { th }}$ on the outage $\varepsilon_{\imath, \text { th }}$ in Fig. 4.3. The derived outage upper bound (using (4.13)) is observed to be tight to the actual simulation curve. 


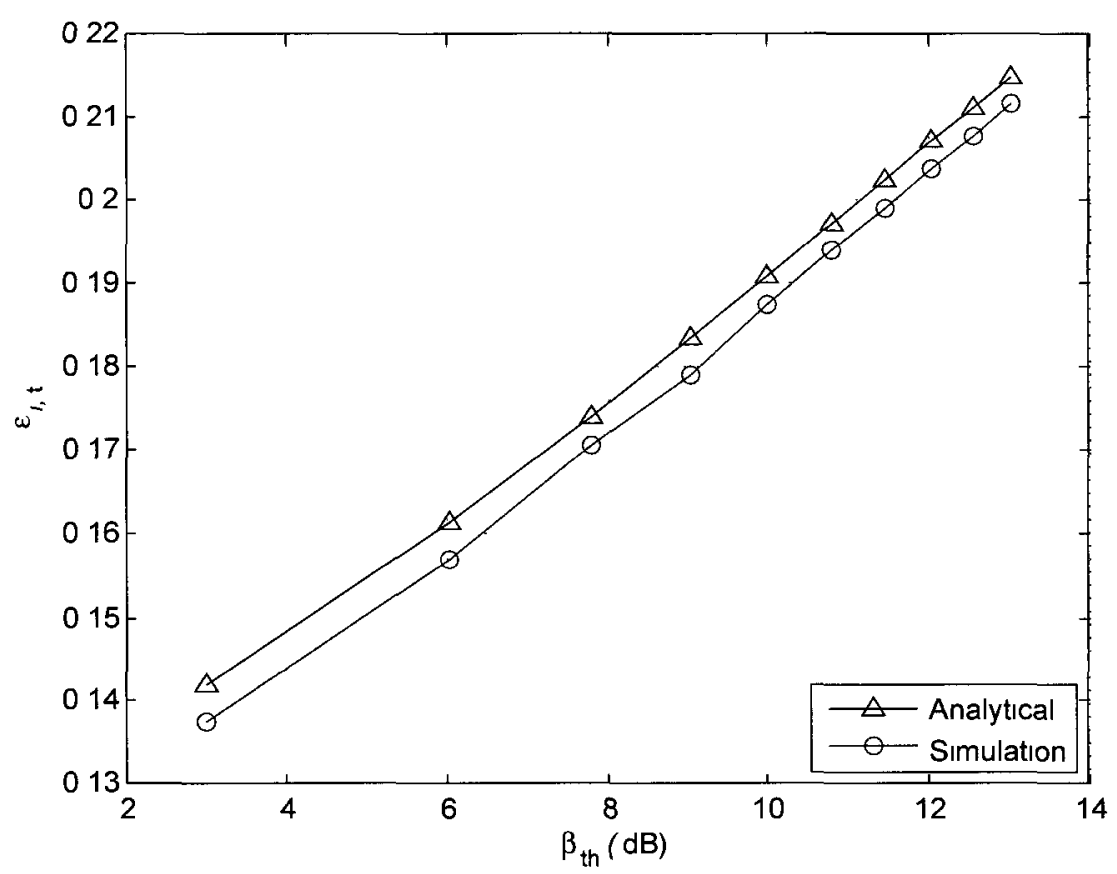

Figure 4.3: Total outage $\varepsilon_{\imath, \mathrm{T}}$ suffered by the $\imath^{\text {th }} \mathrm{PR}$ with the decision threshold $\beta_{\imath, \mathrm{th}}=\beta_{\mathrm{th}}$, for $\lambda=10^{-4} / \mathrm{m}^{2}, \varepsilon_{\imath}=0.05, \alpha=3, \rho_{\imath, \mathrm{r}}=1 \mu \mathrm{W}, \beta_{\imath}=10, \rho_{\mathrm{b}}=1$, and $\rho_{\mathrm{t}}=1 \mathrm{~mW}$. Distance between the adjacent BSs is taken as $500 \mathrm{~m}$.

\subsection{Comparison between Presumably Exact and Soft Exclu- sion Regions by considering no fading and fading, respec- tively on the sensing channel}

It would be interesting to compare the outages and the spectrum sharing gains (number of STs) obtained by using the presumably exact exclusion region formed by assuming no fading and the soft exclusion region formed by taking fading on the sensing channel. We compare the outages and the gains obtained in each of these cases.

Firstly, we obtain difference in the outage values $\delta \varepsilon_{\mathrm{T}}$ for the both cases. In case of no fading, we use the presumably exact exclusion radius $r_{1}=\left(\frac{\rho_{b}}{\eta \beta_{\mathrm{th}}}\right)^{\frac{1}{\alpha}}$ and the same underlying HPPP from which both the processes are derived, i.e., HPPP with exclusion region and HPPP with sensing mechanism, are formed. We plot the $\delta \varepsilon_{\mathbf{T}}$ w.r.t to field size $r_{2}$ in Fig. 4.4 for different values of $\lambda$. We observe that the outage 


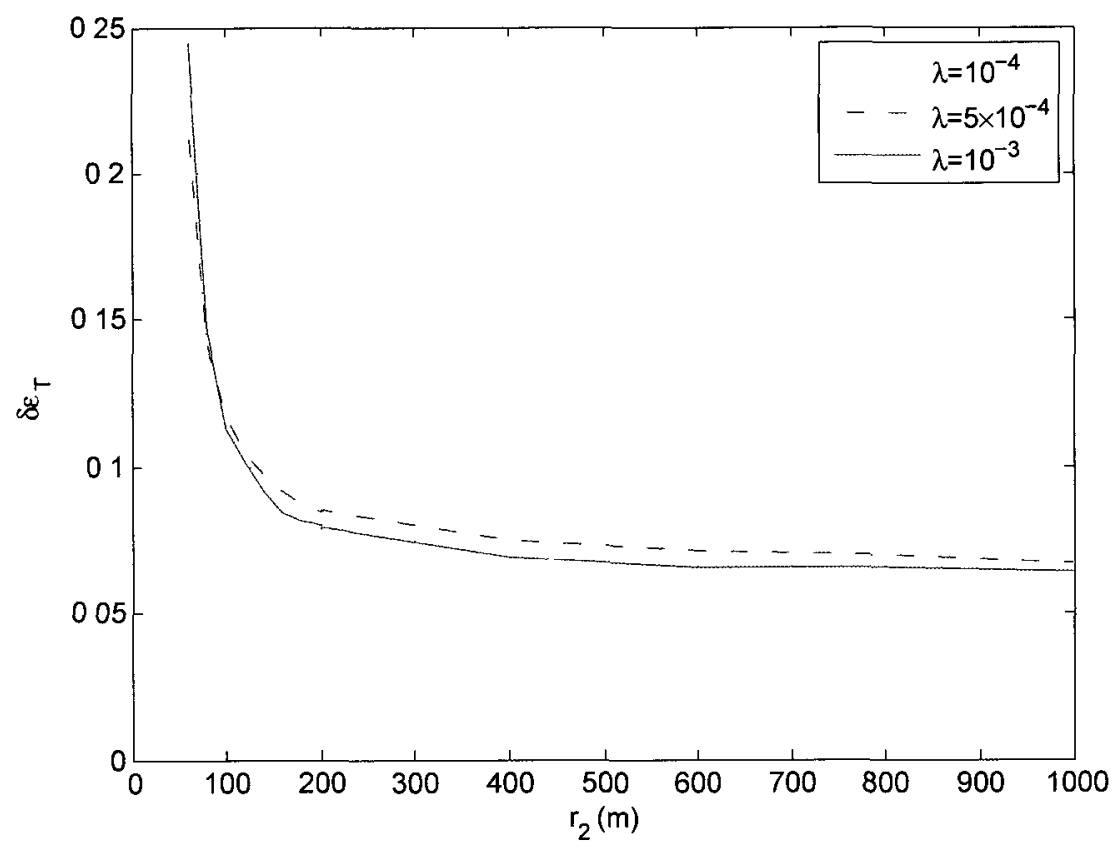

Figure 4.4: Outage difference $\delta \varepsilon_{\mathrm{T}}$ with the field-size $r_{2}$ for different density $\lambda$ values of STs, $\alpha=3, \varepsilon_{1}=0.05, \rho_{\mathrm{t}}=1 \mathrm{~mW}, \beta_{\mathrm{th}}=10$, and $\rho_{\mathrm{b}}=1 \mathrm{~W}$.

in case of presumably exclusion region is always greater than that in case of exclusion region formed by taking fading on the sensing channel. Similar observation is made when we plot the $\delta \varepsilon_{\mathrm{T}}$ w.r.t to $\lambda$ in Fig. 4.5 for different values of field size $r_{2}$. Hence, we conclude that the soft exclusion region formed by taking fading on sensing channel gives lesser outage than using the presumably exact exclusion region.

Let the spectrum sharing gains (expressed in terms of active number of STs) corresponding to the exclusion region mechanism and the sensing mechanism be $N_{1}$ and $N_{2}$ respectively, given by

$$
\begin{aligned}
& N_{1}=\sum_{X(r, \theta) \in \Pi_{\imath, \mathrm{s}}} 1{ }{ }_{\left.r \geq\left(\frac{\rho_{\mathrm{b}}}{\eta \beta_{\mathrm{th}}}\right)^{\frac{1}{\alpha}}\right\}}, \\
& N_{2}=\sum_{X(r, \theta) \in \Pi_{\imath, \mathrm{s}}} \mathbf{1}_{\left\{r \geq\left(\frac{\rho_{\mathrm{b}} \Gamma}{\eta \beta_{\mathrm{th}}}\right)^{\frac{1}{\alpha}}\right\}} .
\end{aligned}
$$




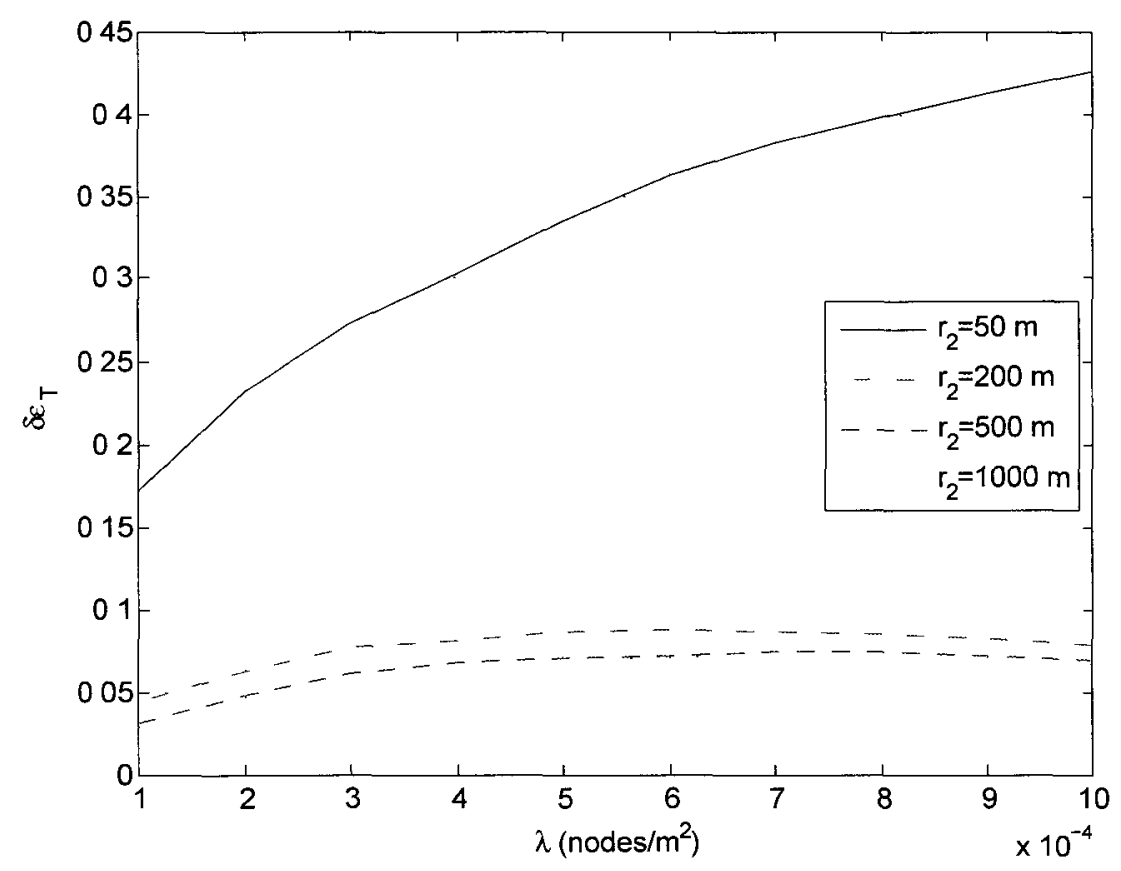

Figure 4.5: Outage difference $\delta \varepsilon_{\mathrm{T}}$ with the density $\lambda$ of STs for different field-size $r_{2}$, $\alpha=3, \varepsilon_{1}=0.05, \rho_{\mathrm{t}}=1 \mathrm{~mW}, \beta_{\mathrm{th}}=10$, and $\rho_{\mathrm{b}}=1 \mathrm{~W}$.

Using (4.9) to obtain the mean $\mu_{2}$ of $N_{2}$ :

$$
\mu_{2}=\mathbb{E}\left(N_{2}\right)=\int_{0}^{2 \pi} \int_{0}^{r_{2}} \lambda_{i, \mathrm{~s}, \mathrm{t}}^{\prime}(r) r \mathrm{~d} r \mathrm{~d} \theta=\int_{0}^{2 \pi} \int_{0}^{r_{2}} \mathcal{P}\left(\frac{\gamma \rho_{\mathrm{b}}}{\eta r^{\alpha}} \leq \beta_{\mathrm{th}}\right) \lambda r \mathrm{~d} r \mathrm{~d} \theta
$$

After some manipulations we get

$$
\mu_{2}=\lambda \pi\left(r_{2}^{2}-\frac{2}{\alpha}\left(\frac{\rho_{\mathrm{b}}}{\eta \beta_{\mathrm{th}}}\right)^{\frac{2}{\alpha}} \gamma\left(\frac{2}{\alpha}, \frac{\eta \beta_{\mathrm{th}} r_{2}^{\alpha}}{\rho_{\mathrm{b}}}\right)\right)
$$

Similarly, it is easy to show $N_{1}$ follows Poisson distribution with mean $\mu_{1}=$ $\lambda \pi\left(r_{2}^{2}-r_{1}^{2}\right)$.

For $r_{2}>r_{1}$ and $\alpha>2$, and after some manipulations, we find that $\mu_{2}>\mu_{1}$. Now, using the cumulative distribution function of the Poisson distribution, we conclude 


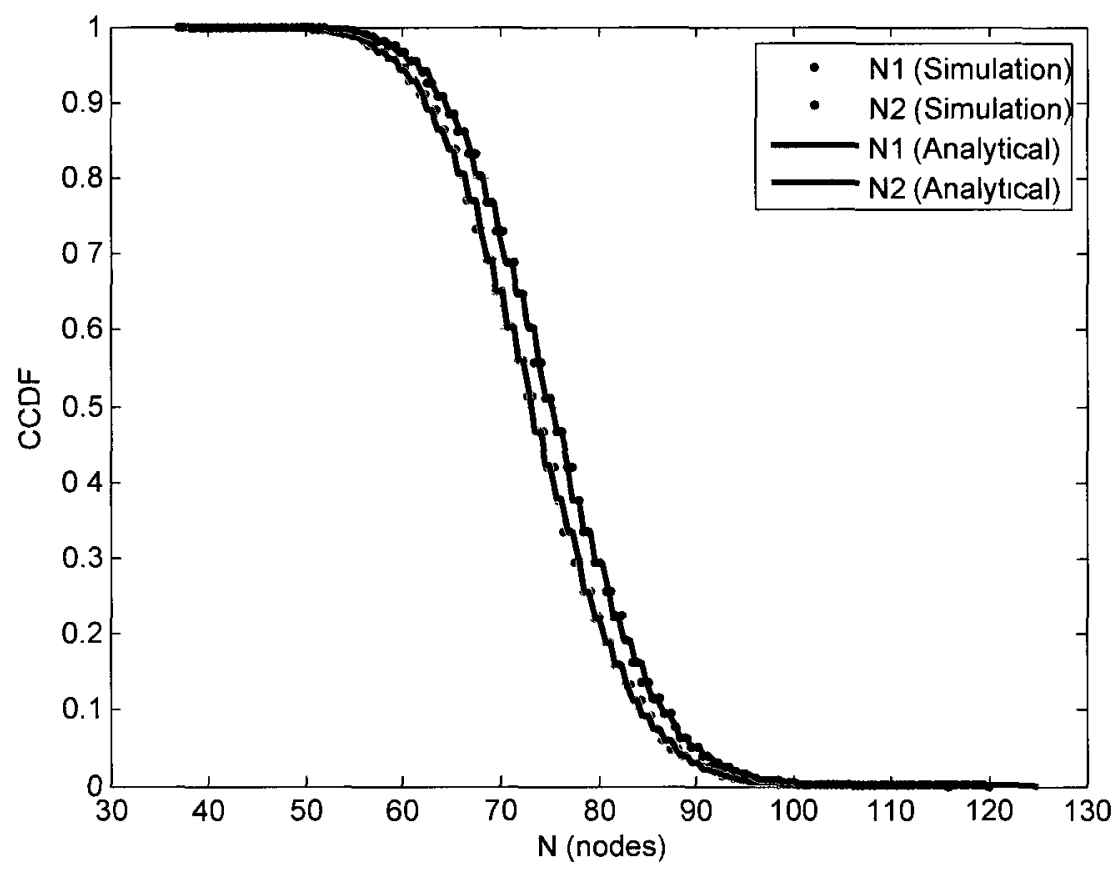

Figure 4.6: CCDF of the number of ST nodes in case of presumably exact exclusion region $N_{1}$ and the soft exclusion region (formed by considering fading on the sensing channel) $N_{2}$, for $r_{2}=100, \eta=10^{-6}, \alpha=3, \beta_{\mathrm{th}}=10$, and $\rho_{\mathrm{b}}=1 \mathrm{~W}$.

that

$$
\mathcal{P}\left(N_{2}>N\right)>\mathcal{P}\left(N_{1}>N\right) \quad \forall N>0 .
$$

We observe this behaviour by plotting CCDF of $N_{1}$ and $N_{2}$ in Fig. 4.6.

Hence, we come up with the interesting results which indicate that for the case of soft exclusion region formed by considering fading on the sensing channel, outage is lower and gain is higher than that of the presumably exact exclusion region. Not only does the fading in the sensing channel give lower outage but also it gives higher spectrum sharing gains. Alternatively, we can also say that Rayleigh fading on the sensing channel to form soft exclusion region helps in achieving better spectrum sharing gains under given outage constraint. Therefore, the previous results on presumably exact exclusion region with Rayleigh fading in their system model, give pessimistic results on the spectrum sharing gains. 


\subsection{Summary}

We have considered a spectrum sharing scenario where a secondary ad hoc network is overlaid on the cellular network, for the uplink case. The PRs in the cellular network are randomly but not necessarily independently distributed. The outage experienced by the cellular receiver nodes is analyzed with and without the sensing mechanism. We derived the closed-form expression for the outage incorporating both sensing and random distribution of nodes (4.13). We make use of the fact that the cellular PRs (or, base-stations) are inherently separated by relatively larger distances to derive a tight upper bound on the outage in case of sensing mechanism. A significant decrease in the outage of the primary receiver is shown, when the sensing mechanism is used to avoid the profound effect of the nearby interferers (STs). Further, we optimized the decision threshold, used by the STs for deciding whether to transmit or not, to maximize the spectrum sharing gains under the given outage constraint. The outage result obtained in the case of sensing mechanism for the PRs can be thought of as pessimistic, however, is also quite safe as there can be sensing errors due to the hardware inaccuracies [4]. The upper bound derived here gives an assurance that even if some sensing errors happen, the primary services do not suffer beyond the upper bound on the outage. We also made an interesting observation that when the soft exclusion region (formed by considering fading on the sensing channel) is preferred over the presumably exact exclusion region, not only the lower outage but also the higher spectrum sharing gains are achieved. 


\section{Chapter 5}

\section{Conclusion and Future Work}

\subsection{Thesis Achievements}

The presented work provides a preliminary basis upon which a secondary network can be deployed to share the spectrum of a primary network. Network operators can get the insights on the spectrum sharing gains and the degradation of primary services to determine the feasibility of the spectrum sharing. It should be noted that the derived closed-form results can be used for the outage experienced by any typical victim receiver due to finite field of interferers, in general. Similarly, the closedform results incorporating the sensing mechanism can be extended, in general, to any set of spatially distributed interferers. We confirm the analytical results obtained by comparing the results with the Monte Carlo simulations. We summarize our conclusions as follows:

- In Chapter 2, we made an interesting observation on the outage that any receiver experiences due to two independent unwanted signal sets, without the need for the knowledge of the distribution of the net unwanted signal from one of these sets; only the outage resulting due to this set is needed. This result is widely applicable where the distribution of the net unwanted signal from one of the independent unwanted signal set is unknown or is hard to characterize. Futher 
the result can be extended to any number of unwanted signal sets with known outages. We also conclude that the additional outage suffered by a victim receiver due to new unwanted signal set is lower when the initial outage is higher.

- In Chapter 3, we showed the significance of the derived equation in the spectrum sharing scenario where it helps in getting rid of the herculean task of characterizing the interference coming from the primary network. We observed that the initial outage at the victim receiver plays an important role in determining the compatibility of primary network with the secondary network which has been ignored in the past works. It also helps in determining what kind of networks are suitable for the spectrum sharing so that significant gains can be achieved for secondary network within the specified outage constraint.

- We derived closed-form expressions for the outage and the spectrum sharing gains (in terms of average no. of STs) when the $\mathrm{SN}$ is distributed over a finite region. Along with that various system parameters are analyzed and reflected in the form of closed-form results. We study the spectrum sharing opportunities and gains through closed-form expressions under the given framework. Moreover, we compare the outages obtained from the singular and non-singular path loss models and showed that the difference in the outage values obtained from both the cases is quite insignificant.

- We studied the uplink case of the cellular network overlaid by the ad hoc network in Chapter 3. Unlike previous works, here the cellular receivers (BSs) can be interacting with each other in complex ways and need not to be independently distributed. Instead of a presumably exact exclusion region (also known as guard zone or avoidance zone), we used sensing mechanism around 
every cellular receiver to alleviate the profound effect of nearby interfering transmissions. Although the overlay of primary cellular network with a secondary ad hoc network has been considered before, the closed-form expression for the outage incorporating both sensing and random distribution of nodes has been derived for the first time (4.13). We exploit the inherent property of the cellular network that the co-channel receivers are spatially separated by relatively larger distances to derive tight upper outage bound. The derived results also accomodate diverse primary receivers where every PR-PT link can be different. Furthermore, the soft exclusion region (formed by considering fading in the sensing channel) is shown to be better than the presumably exact exclusion region (formed by considering no fading on the sensing channel) in providing the lower outage at the primary user as well as the higher spectrum sharing gains.

\subsection{Future Work}

Due to time limitations, we were not able to pursue the possible extensions of this work. Some of the works that we hope to pursue in the future are as follows. Various other design parameters such as the variable transmission power, variable density, irregular field size can be studied using numerical methods under the given framework. In the overlay scheme [3], there is always a possibility of active SUs even in the presence of PU due to spectrum sensing inefficiencies (hardware, fading, shadowing). Some additional factors can be incorporated in this work, accounting these uncertainities, to keep the average behaviour or harm by the SN under regulation.

More comprehensive models of spectrum sharing can be built on this work. In general, the STs can be distributed according to a general point process and if the Laplace transform of the distribution of the interference from such a network can be 
found, we may apply (2.11) to study the additional outage. For example, [47] studied the outage in the case where the typical receiver's transmitter is itself the part of Poisson cluster process (Matern-cluster or Neyman-cluster) in a single network case. They derived the numerically integrable expression for the Laplace transform of the interference distribution which is dependent on the position of the typical receiver. This can be extended to the case of spectrum sharing networks where the STs are deployed as Poisson cluster process over an infinite region. Due to the spatial stationarity property of Poisson cluster process [8], statistics of the secondary interference at every point are the same. Hence, the Laplace transform of the interference distribution at a typical victim receiver node is equal to the Laplace transform of the interference distribution coming from other clusters (i.e., without conditioning cluster at origin) [47, quantity $T_{1}$ in (34)]. We can put that equation in our formulations. 


\section{Bibliography}

[1] "Report of the spectrum efficiency working group," FCC Spectrum Policy Task Force, Nov. 2002.

[2] M. A. McHenry, P. A. Tenhula, D. McCloskey, D. A. Roberson, and C. S. Hood, "Chicago spectrum occupancy measurements \& analysis and a long-term studies proposal," in Proc. of the International Workshop on Technology and Policy for Accessing Spectrum. New York, NY, USA: ACM, 2006, p. 1.

[3] I. Akyildiz, W. Lee, M. Vuran, and S. Mohanty, "Next generation/dynamic spectrum access/cognitive radio wireless networks: a survey," Comp. Networks J., vol. 50, no. 13, pp. $2127-2159$, Sep. 2006.

[4] T. Yucek and H. Arslan, "A survey of spectrum sensing algorithms for cognitive radio applications," IEEE Commun. Surveys Tuts., vol. 11, no. 1, pp. $116-130$, Mar. 2009.

[5] F. Baccelli and B. Blaszczyszyn, "Stochastic geometry and wireless networks: Volume I theory," Found. Trends Netw., vol. 3, pp. 249-449, Mar. 2009, [Online] Available: http://dx.doi.org/10.1561/1300000006.

[6] J. Andrews, R. Ganti, M. Haenggi, N. Jindal, and S. Weber, "A primer on spatial modeling and analysis in wireless networks," IEEE Commun. Mag., vol. 48, no. 11 , pp. $156-163$, Nov. 2010. 
[7] M. Haenggi, J. Andrews, F. Baccelli, O. Dousse, and M. Franceschetti, "Stochastic geometry and random graphs for the analysis and design of wireless networks," IEEE J. Sel. Areas Commun., vol. 27, no. 7, pp. 1029-1046, Sep. 2009.

[8] D. Stoyan, W. Kendell, and J. Mecke, Stochastic Geometry and its Applications, 2nd ed., ser. Wiley Series in Probability and Mathematical Statistics. New York: Wiley, 1995.

[9] M. Win, P. Pinto, and L. Shepp, "A mathematical theory of network interference and its applications," Proc. IEEE, vol. 97, pp. 205-230, Feb. 2009.

[10] E. Sousa and J. Silvester, "Optimum transmission ranges in a direct-sequence spread-spectrum multihop packet radio network," IEEE J. Sel. Areas Commun., vol. 8, no. 5, pp. 762-771, Jun. 1990.

[11] R. Menon, R. Buehrer, and J. Reed, "On the impact of dynamic spectrum sharing techniques on legacy radio systems," IEEE Trans. Wireless Commun., vol. 7, no. 11, pp. 4198-4207, Nov. 2008.

[12] A. Ghasemi and E. Sousa, "Interference aggregation in spectrum-sensing cognitive wireless networks," IEEE J. Sel. Topics Signal Process., vol. 2, no. 1, pp. 41-56, Feb. 2008.

[13] C. C. Chan and S. Hanly, "Calculating the outage probability in a CDMA network with spatial Poisson traffic," IEEE Trans. Veh. Technol., vol. 50, no. 1, pp. $183-204$, Jan. 2001.

[14] M. Aljuaid, "Interference characterization and spectrum sharing in large wireless networks," Ph.D. dissertation, Systems and Computer Department, Carleton University, Ottawa, Canada, May 2010. [Online]. 
Available: http://www.sce.carleton.ca/faculty/yanikomeroglu/Pub/PhDThesisMuhammadAljuaid-ProfYanikomeroglu-CarletonU-August2010.pdf.

[15] M. Vu, N. Devroye, and V. Tarokh, "On the primary exclusive region of cognitive networks," IEEE Trans. Wireless Commun., vol. 8, no. 7, pp. $3380-3385$, Jul. 2009.

[16] R. Dahama, K. Sowerby, and G. Rowe, "Protection regions for dynamic spectrum sharing," Electron. Lett., vol. 46, no. 20, pp. $1407-1408$, Sep. 2010.

[17] M. Aljuaid and H. Yanikomeroglu, "Investigating the Gaussian convergence of the distribution of the aggregate interference power in large wireless networks," IEEE Trans. Veh. Technol., vol. 59, no. 9, pp. 4418-4424, Nov. 2010.

[18] V. Mordachev and S. Loyka, "On node density - outage probability tradeoff in wireless networks," IEEE J. Sel. Areas Commun., vol. 27, no. 7, pp. 1120-1131, Sep. 2009.

[19] M. Haenggi and R. K. Ganti, "Interference in large wireless networks," Found. Trends Netw., vol. 3, pp. 127-248, Feb. 2009.

[20] M. Khoshkholgh, K. Navaie, and H. Yanikomeroglu, "Impact of the secondary network on the outage performance of the primary service in spectrum sharing," in IEEE International Conference on Communications, May 2010.

[21] M. Aljuaid and H. Yanikomeroglu, "Impact of secondary users' field size on spectrum sharing opportunities," in IEEE Wireless Communications and Networking Conference, Apr. 2010.

[22] E. Salbaroli and A. Zanella, "Interference analysis in a Poisson field of nodes of finite area," IEEE Trans. Veh. Technol., vol. 58, no. 4, pp. 1776-1783, May 2009 . 
[23] A. Hasan and J. Andrews, "The guard zone in wireless ad hoc networks," IEEE Trans. Wireless Commun., vol. 6, no. 3, pp. 897-906, Mar. 2007.

[24] J. Venkataraman, M. Haenggi, and O. Collins, "Shot noise models for outage and throughput analyses in wireless ad hoc networks," in IEEE Miltary Communıcations Conference, Oct. 2006.

[25] V. Chandrasekhar and J. Andrews, "Uplink capacity and interference avoidance for two-tier femtocell networks," IEEE Trans. Wireless Commun., vol. 8, no. 7, pp. 3498-3509, Jul. 2009.

[26] K. Huang, V. Lau, and Y. Chen, "Spectrum sharing between cellular and mobile ad hoc networks: transmission-capacity trade-off," IEEE J. Sel. Areas Commun., vol. 27 , no. 7 , pp. 1256-1267, Sep. 2009.

[27] S. Weber, J. G. Andrews, and N. Jindal, "An overview of the transmission capacity of wireless networks," IEEE Trans. Commun., vol. 58, no. 12, pp. 3593-3604, Dec. 2010.

[28] H. Wu, D. Li, and C. Yin, "Transmission capacities for overlaid spread-spectrum wireless networks with Rayleigh channel," in Network Infrastructure and Dıgital Content, 2009. IC-NIDC 2009. IEEE International Conference on, Nov. 2009, pp. 89-94.

[29] J. Kingman, Pozsson Processes. Oxford University Press US, 1993.

[30] A. Iyer, C. Rosenberg, and A. Karnik, "What is the right model for wireless channel interference?" IEEE Trans. Wireless Commun., vol. 8, no. 5, pp. 2662 -2671 , May 2009.

[31] Q. Zhao and B. Sadler, "A survey of dynamic spectrum access," IEEE Srgnal Process. Mag., vol. 24, no. 3, pp. 79-89, May 2007. 
[32] S. Zaidi, D. McLernon, and M. Ghogho, "Outage probability analysis of cognitive radio networks under self-coexistence constraint," in Annual Conference on Information Scıences and Systems, Mar. 2010.

[33] R. Dahama, K. Sowerby, and G. Rowe, "Outage probability estimation for licensed systems in the presence of cognitive radio interference," in IEEE Vehıcular Technology Conference, Apr. 2009.

[34] M. Timmers, S. Pollin, A. Dejonghe, A. Bahai, L. Van der Perre, and F. Catthoor, "Accumulative interference modeling for cognitive radios with distributed channel access," in International Conference on Cognitıve Radıo Ortented Wireless Networks and Communications, May 2008.

[35] Z. Chen, C.-X. Wang, X. Hong, J. Thompson, S. A. Vorobyov, X. Ge, H. Xiao, and F. Zhao, "Aggregate interference modeling in cognitive radio networks with power and contention control," submitted to IEEE Trans. Commun., Jul. 2010, [Online] Available: http://arxiv.org/abs/1008.1043.

[36] M. Vu, N. Devroye, and V. Tarokh, "The primary exclusive region in cognitive networks," in IEEE Consumer Communıcations and Networking Conference, Jan. 2008, pp. 1014-1019.

[37] C. Yin, C. Chen, T. Liu, and S. Cui, "Generalized results of transmission capacities for overlaid wireless networks," in IEEE Internatıonal Symposıum on Informatıon Theory, Jun. 2009, pp. 1774-1778.

[38] L. Lee, J. Andrews, and D. Hong, "Spectrum-sharing transmission capacity," submitted to IEEE Trans. Wireless Commun., Nov. 2010, [Online] Available: http://wncg.org/publications/dl.php?file=Andrews1288631643.pdf. 
[39] M. Aljuaid and H. Yanikomeroglu, "Identifying boundaries of dominant regions dictating spectrum sharing opportunities for large secondary networks," in $I E E E$ Internatıonal Symposium on Personal Indoor and Mobıle Radıo Communıcatrons, Sep. 2010.

[40] I. S. Gradshteyn and I. M. Ryzhik, Tables of Integrals, Sernes, and Products, 7th ed. Academic Press, 2007.

[41] A. Ghasemi, "Statistical characterization of interference in cognitive radio networks," in IEEE Internatıonal Symposium on Personal, Indoor and Mobıle Radıo Communications, Sep. 2008.

[42] R. Menon, R. M. Buehrer, and J. H. Reed, "Impact of exclusion region and spreading in spectrum-sharing ad hoc networks," in Proc. of the International Workshop on Technology and Polncy for Accessing Spectrum. New York, NY, USA: ACM, 2006, p. 7.

[43] M. Aljuaid and H. Yanikomeroglu, "A cumulant-based investigation of the impact of secondary users' field size on spectrum sharing opportunities," accepted to IEEE Trans. Veh. Technol., May 2011.

[44] H. Inaltekin, S. Wicker, M. Chiang, and H. Poor, "On unbounded path-loss models: effects of singularity on wireless network performance," IEEE J. Sel. Areas Commun., vol. 27, no. 7, pp. 1078 -1092, Sep. 2009.

[45] M. Haenggi and D. Puccinelli, "Routing in ad hoc networks: a case for long hops," IEEE Commun. Mag., vol. 43, no. 10, pp. 93 - 101, Oct. 2005.

[46] C. han Lee and M. Haenggi, "Interference and outage in doubly Poisson cognitive networks," in Proc. International Conference on Computer Communications and Networks, Aug. 2010. 
[47] R. Ganti and M. Haenggi, "Interference and outage in clustered wireless ad hoc networks," IEEE Trans. Inf. Theory, vol. 55, no. 9, pp. 4067-4086, Sep. 2009. 